\title{
Boussinesq modeling of surface waves due to underwater landslides
}

\author{
D. Dutykh ${ }^{1,2}$ and H. Kalisch ${ }^{3}$ \\ ${ }^{1}$ University College Dublin, School of Mathematical Sciences, Belfield, Dublin 4, Ireland \\ ${ }^{2}$ LAMA, UMR5127, CNRS - Université de Savoie, Campus Scientifique, 73376 Le Bourget-du-Lac Cedex, France \\ ${ }^{3}$ Department of Mathematics, University of Bergen, P.O. Box 7800, 5020 Bergen, Norway
}

Correspondence to: D. Dutykh (denys.dutykh@ucd.ie)

Received: 14 November 2012 - Revised: 8 April 2013 - Accepted: 9 April 2013 - Published: 3 May 2013

\begin{abstract}
Consideration is given to the influence of an underwater landslide on waves at the surface of a shallow body of fluid. The equations of motion that govern the evolution of the barycenter of the landslide mass include various dissipative effects due to bottom friction, internal energy dissipation, and viscous drag. The surface waves are studied in the Boussinesq scaling, with time-dependent bathymetry. A numerical model for the Boussinesq equations is introduced that is able to handle time-dependent bottom topography, and the equations of motion for the landslide and surface waves are solved simultaneously.

The numerical solver for the Boussinesq equations can also be restricted to implement a shallow-water solver, and the shallow-water and Boussinesq configurations are compared. A particular bathymetry is chosen to illustrate the general method, and it is found that the Boussinesq system predicts larger wave run-up than the shallow-water theory in the example treated in this paper. It is also found that the finite fluid domain has a significant impact on the behavior of the wave run-up.
\end{abstract}

\section{Introduction}

Surface waves originating from sudden perturbations of the bottom topography are often termed tsunamis. Two distinct generation mechanisms of a tsunami are underwater earthquakes and submarine mass failures. Among the broad class of submarine mass failures, landslides can be characterized as translational failures that travel considerable distances along the bottom profile (Grilli and Watts, 2005; Prior and Coleman, 1979). In the past, the role of landslides and rockfalls in the excitation of tsunamis may have been underestimated, as most known occurrences of tsunamis were accredited to seismic activity. However, it is now more accepted that submarine mass failures also contribute to a large portion of tsunamis (Tinti et al., 2001), and recent years have seen a multitude of works devoted to the study of such underwater landslides and the resulting effect on surface waves (Bardet et al., 2003; Chubarov et al., 2011; Didenkulova et al., 2010; Fernandez-Nieto et al., 2008; Grilli and Watts, 1999, 2005; Okal, 2003; Okal and Synolakis, 2003; Poncet et al., 2010; Tinti et al., 2001). As suggested in Fritz et al. (2007), it is possible for underwater landslides and earthquakes to act in tandem, and produce very large surface waves

A natural question to ask is whether the effect of underwater landslides on surface waves can be such that they may pose a danger for civil engineering structures located near the shore. Consequently, one important issue is the wave action and in particular the run-up and drawdown at beaches in the vicinity of the landslide. While the drawdown itself may not pose a threat, one consequence of a large drawdown can be the amplification of the run-up of the following positive wave crest (Dutykh et al., 2011a; Tadepalli and Synolakis, 1996).

There have been many numerical and a few experimental studies devoted to this subject, but it is generally difficult to include many of the complex parameters and dependencies of a realistic landslide into a physical model. Therefore, most workers attempt to distill the problem to a model setup where many effects such as turbulence and sedimentation are disregarded. For example, Grilli and Watts (2005) study tsunami sensitivity to several landslide parameters in the case of a landslide in a coastal area of an open ocean. In particular, dependence on the landslide shape and the initial depth of the landslide location are studied, and it is found that the landslide with the smallest length produced the largest wave height and run-up, and that the wave run-up at an adjacent 
beach is inversely proportional to the initial depth. The work in Grilli and Watts (2005) relies on integrating the full waterwave equations using an irrotational boundary-element code, and using an open boundary with transmission conditions (Grilli et al., 2001, 2010). While most works have considered a given dynamics for the landslide, the bottom motion in Grilli and Watts (2005) is described by an ordinary differential equation similar to the one used here. Thus the motion of the landslide is computed using a differential equation derived from first principles using Newtonian mechanics. However to expedite comparison with experiments, the landslide in Grilli and Watts (2005) is considered to have moved on a straight inclined bottom with constant slope.

More recently, Khakimzyanov and Shokina (2010) and Chubarov et al. (2011) have also used a differential equation to find the bottom motion. One major novelty in their work is that the landslide motion is computed on a bottom with an arbitrary shape. The time-dependent bathymetry is then used to drive a numerical solver of the shallow-water equations. An advantage of this approach when compared to Grilli and Watts (2005) is the reduced computation time. On the other hand, the description of the wave motion in the shallow-water theory is only approximate, and in particular, one important effect of surface waves, namely the influence of frequency dispersion is neglected.

The main aim of the current work is to study the dispersive wave generation in a closed basin (Beisel et al., 2012) using a more realistic landslide model (Chubarov et al., 2005) while keeping the simplicity of the shallow-water approach. To this end, we use the so-called Peregrine system, which is a particular case of a general class of model systems that arise in the Boussinesq scaling (Boussinesq, 1871). A common feature of all Boussinesq-type systems is that they allow a simplified study of surface waves in which both nonlinear and dispersive effects are taken into account. In the present case, we need to use a Boussinesq system that can handle complex and time-dependent bottom topography. Such a system was derived by $\mathrm{Wu}$ (1987), and can be used in connection with the dynamic bathymetry. An example of the type of situation considered here is shown in Fig. 1, which shows how the bathymetry is given by the combination of a solid bottom, and a landslide profile sliding along the fixed bottom.

We conduct two main experiments. First, a comparison with the shallow-water theory is carried out. Second, the dependence of the tsunami characteristics on the initial depth of the landslide is investigated. The main findings of the present work are that the predictions of the shallow-water and Boussinesq theory are divergent for the cases treated in this paper, and that the effect of a finite fluid domain, such as a river, lake or fjord (Poncet et al., 2010), can lead to significantly different behavior when compared to tsunamis on an open ocean (see also Beisel et al., 2012).

The Boussinesq model in this paper is based on the assumption of an inviscid fluid, and irrotational flow. These are standard assumptions in the study of surface waves, and

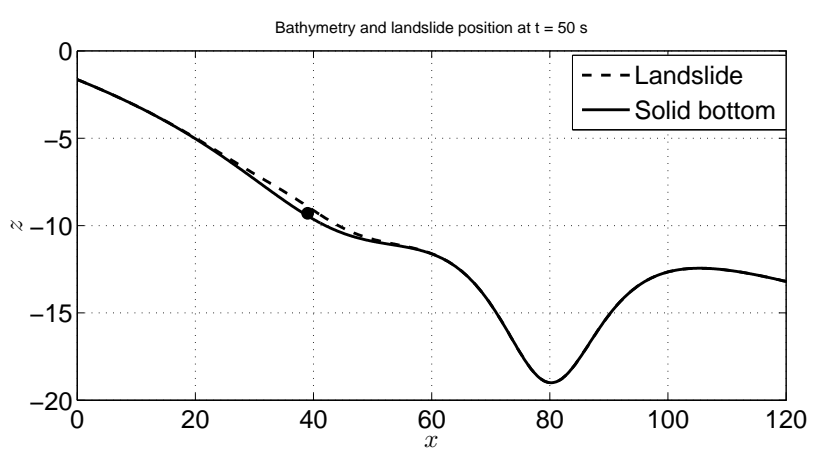

Fig. 1. The fixed bathymetry $z=h_{0}(x)$ and the position of the landslide after $50 \mathrm{~s}$. The position of the barycenter is indicated by a black dot.

generally give good results, unless there are strong background currents in the fluid. Another effect that is not taken account of here is the wave resistance on the landslide due to waves created by the motion of the landslide. However, as observed in Harbitz et al. (2006), this effect is negligible for most realistic cases of underwater landslides. Viscosity is included in the dynamic model for the landslide as will be shown in the next section. In order to capture the effect of slide deformation during the evolution, a damping term in the equation of motion is included to model the internal friction in the landslide mass.

The paper is organized in the following way. In Sect. 2, the equation of motion for the landslide is developed. Then in Sect. 3, the Boussinesq model is recalled. In Sect. 4, solitarywave solutions of the Peregrine system are found numerically. In Sect. 5, the numerical scheme for the Boussinesq system is explained and the numerical method is tested using the exact solutions of Sect. 4. Section 6 contains results of numerical runs for a few specific cases of bottom bathymetry, a parameter study of wave run-up in relation to the initial depth of the landslide, and a comparison with the shallowwater theory.

\section{The landslide model}

In this section we briefly present a mathematical model of underwater landslide motion. This process has to be addressed carefully since it determines the subsequent formation of water waves at the free surface. In the present study, we will assume the movable mass to be a solid body with a prescribed shape and known physical properties. Since the landslide mass and volume is preserved during the evolution, it is sufficient to determine the position of the barycenter $x=x_{\mathrm{c}}(t)$ as a proxy for the motion of the whole body. As observed in the introduction, most studies of wave generation due to underwater landslides are based on prescribed bottom motion, or on solving the equation of motion on a uniform slope while taking account of different types of friction and 
viscous terms. Examples of such works include the following: DiRisio et al. (2009); Pelinovsky and Poplavsky (1996); Watts et al. (2000). A more general approach was recently pioneered by Khakimzyanov and Shokina (2010), where curvature effects of the bottom topography were taken into account. Since this model is applicable to a wider range of cases, we follow the approach of Khakimzyanov and Shokina (2010). However, in addition to the effects included by Khakimzyanov and Shokina, our model also incorporates the effect of internal friction in the slide material (given by the dissipative force $F_{\mathrm{i}}$ ) and the action of bottom friction, given by $F_{\mathrm{b}}$.

The static bathymetry is prescribed by a sufficiently smooth single-valued function $z=-h_{0}(x)$, and the landslide shape is initially prescribed by a localized function $z=\zeta_{0}(x)$. To be specific, in this study we choose the following shape function for the landslide mass:

$\zeta_{0}(x)=A \begin{cases}\frac{1}{2}\left(1+\cos \left(\frac{2 \pi\left(x-x_{0}\right)}{\ell}\right)\right), & \left|x-x_{0}\right| \leq \frac{\ell}{2} \\ 0, & \left|x-x_{0}\right|>\frac{\ell}{2} .\end{cases}$

In this formula, $A$ is the maximum height, $\ell$ the length of the slide and $x_{0}$ the initial position of its barycenter. It is clear that the model description given below and the method of numerical integration used in the present work is applicable to any other smooth profile, as long as it is sufficiently localized and fully submerged.

Since the landslide motion is translational, its shape at time $t$ is given by the function $z=\zeta(x, t)=\zeta_{0}\left(x-x_{\mathrm{c}}(t)\right)$. Recall that the landslide center is located at a point with abscissa $x=x_{\mathrm{c}}(t)$. Then, the impermeable bottom for the water wave problem can be easily determined at any time by simply superposing the static and dynamic components. Thus the bottom boundary conditions for the fluid are to be imposed at

$z=-h(x, t)=-h_{0}(x)+\zeta(x, t)$.

To simplify the subsequent presentation, we introduce the classical arc-length parameterization, where the parameter $s=s(x)$ is given by the formula

$s=L(x)=\int_{x_{0}}^{x} \sqrt{1+\left(h_{0}^{\prime}(\xi)\right)^{2}} \mathrm{~d} \xi$.

The function $L(x)$ is monotone and can be efficiently inverted to yield the original Cartesian abscissa $x=L^{-1}(s)$. Within the parameterization in Eq. (2), the center of the landslide is initially located at a point with the curvilinear coordinate $s=0$. The local tangential direction is denoted by $\tau$ and the normal direction by $n$.

A straightforward application of Newton's second law reveals that the landslide motion is governed by the differential equation

$m \frac{\mathrm{d}^{2} s}{\mathrm{~d} t^{2}}=F_{\tau}(t)$ where $m$ is the landslide mass and $F_{\tau}(t)$ is the tangential component of the sum of forces acting on the moving submerged body. In order to project the forces onto the axes of the local coordinate system, the angle $\theta(x)$ between $\tau$ and $O x$ is needed. This angle is determined by

$\theta(x)=-\arctan \left(h_{0}^{\prime}(x)\right)$.

Let us denote by $\rho_{\mathrm{w}}$ and $\rho_{\ell}$ the densities of the water and landslide material correspondingly. If $V$ is the volume of the slide, then the total mass $m$ is given by the expression

$m=\left(\rho_{\ell}+c_{\mathrm{w}} \rho_{\mathrm{w}}\right) V$,

where $c_{\mathrm{W}}$ is the added mass coefficient. As explained in Batchelor (2000), a portion of the water mass has to be added to the mass of the landslide since it is entrained by the underwater body motion. For a cylinder, the coefficient $c_{\mathrm{w}}$ is equal exactly to one, but in the present case, the coefficient has to be estimated. The volume of the sliding material is given by $V=W \cdot S$, where $W$ is the landslide width in the transverse direction, and $S$ can be computed by

$S=\int_{\mathbb{R}} \zeta_{0}(x) \mathrm{d} x$.

The last integral can be computed exactly for the particular choice in Eq. (1) of the landslide shape to give

$V=\frac{1}{2} \ell A W$.

The total projected force acting on the landslide can be conventionally represented as a sum of the force $F_{\mathrm{g}}$ representing the joint action of gravity and buoyancy, and the total contribution of various dissipative forces.

The gravity and buoyancy forces act in opposite directions, and their horizontal projection $F_{\mathrm{g}}$ can be easily computed by

$F_{\mathrm{g}}(t)=\left(\rho_{\ell}-\rho_{\mathrm{w}}\right) W g \int_{\mathbb{R}} \zeta(x, t) \sin (\theta(x)) \mathrm{d} x$.

Now, let us specify the dissipative forces. The water resistance to the motion of the landslide $F_{\mathrm{r}}$ due to viscous dissipation is proportional to the maximal transverse section of the moving body and to the square of its velocity. In addition, the coefficient sign $\left(\frac{\mathrm{d} s}{\mathrm{~d} t}\right)$ is needed to dissipate the landslide kinetic energy independently of its direction of motion. Thus the force $F_{\mathrm{r}}$ takes the form

$$
F_{\mathrm{r}}=-\operatorname{sign}\left(\frac{\mathrm{d} s}{\mathrm{~d} t}\right) \frac{1}{2} c_{\mathrm{d}} \rho_{\mathrm{w}} A W\left(\frac{\mathrm{d} s}{\mathrm{~d} t}\right)^{2},
$$

where $c_{\mathrm{d}}$ is the resistance coefficient of the water. The friction force $F_{\mathrm{f}}$ is proportional to the normal force exerted on the body due to the weight:

$F_{\mathrm{f}}=-c_{\mathrm{f}} \operatorname{sign}\left(\frac{\mathrm{d} s}{\mathrm{~d} t}\right) N(x, t)$. 
The normal force $N(x, t)$ is composed not only of the normal components of gravity and buoyancy forces, but also of the centripetal force due to the variation of the bottom slope:

$$
\begin{aligned}
N(x, t) & =\left(\rho_{\ell}-\rho_{\mathrm{w}}\right) g W \int_{\mathbb{R}} \zeta(x, t) \cos (\theta(x)) \mathrm{d} x \\
& +\rho_{\ell} W \int_{\mathbb{R}} \zeta(x, t) \kappa(x)\left(\frac{\mathrm{d} s}{\mathrm{~d} t}\right)^{2} \mathrm{~d} x .
\end{aligned}
$$

Here $\kappa(x)$ is the signed curvature of the bottom, which can be computed using the formula

$\kappa(x)=\frac{h_{0}^{\prime \prime}(x)}{\left(1+\left(h_{0}^{\prime}(x)\right)^{2}\right)^{\frac{3}{2}}}$.

We note that the last term vanishes for a plane bottom since $\kappa(x) \equiv 0$ in this particular case. Energy loss inside the sliding material due to internal friction is modeled by

$$
F_{\mathrm{i}}=-c_{\mathrm{v}} \rho_{\ell} W S \frac{\mathrm{d} s}{\mathrm{~d} t},
$$

where $c_{\mathrm{V}}$ is an internal friction coefficient. Finally, dissipation in the boundary layer between the landslide and the solid bottom is taken account of by the term

$$
F_{\mathrm{b}}=-c_{\mathrm{b}} \rho_{\mathrm{w}} W \ell \frac{\mathrm{d} s}{\mathrm{~d} t}\left|\frac{\mathrm{d} s}{\mathrm{~d} t}\right|,
$$

where $c_{\mathrm{b}}$ is the Chézy coefficient.

Finally, if we sum up the contributions of all the forces described above, we obtain the second-order differential equation

$$
\begin{aligned}
\left(\gamma+c_{\mathrm{w}}\right) S \frac{\mathrm{d}^{2} s}{\mathrm{~d} t^{2}}= & (\gamma-1) g\left(\mathcal{I}_{1}(t)-c_{\mathrm{f}} \sigma(t) \mathcal{I}_{2}(t)\right) \\
& -\sigma(t)\left(c_{\mathrm{f}} \gamma \mathcal{I}_{3}(t)+\frac{1}{2} c_{\mathrm{d}} A\right)\left(\frac{\mathrm{d} s}{\mathrm{~d} t}\right)^{2} \\
& -c_{\mathrm{v}} \gamma S \frac{\mathrm{d} s}{\mathrm{~d} t}-c_{\mathrm{b}} \ell \frac{\mathrm{d} s}{\mathrm{~d} t}\left|\frac{\mathrm{d} s}{\mathrm{~d} t}\right|,
\end{aligned}
$$

where $\gamma=\frac{\rho_{\ell}}{\rho_{\mathrm{w}}}>1$ is the ratio of densities, $\sigma(t)=\operatorname{sign}\left(\frac{\mathrm{d} s}{\mathrm{~d} t}\right)$ and the integrals $\mathcal{I}_{1,2,3}(t)$ are defined by

$$
\begin{aligned}
& \mathcal{I}_{1}(t)=\int_{\mathbb{R}} \zeta(x, t) \sin (\theta(x)) \mathrm{d} x, \\
& \mathcal{I}_{2}(t)=\int_{\mathbb{R}} \zeta(x, t) \cos (\theta(x)) \mathrm{d} x, \\
& \mathcal{I}_{3}(t)=\int_{\mathbb{R}} \zeta(x, t) \kappa(x) \mathrm{d} x .
\end{aligned}
$$

In order to obtain a well-posed initial value problem, Eq. (4) has to be supplemented with initial conditions for
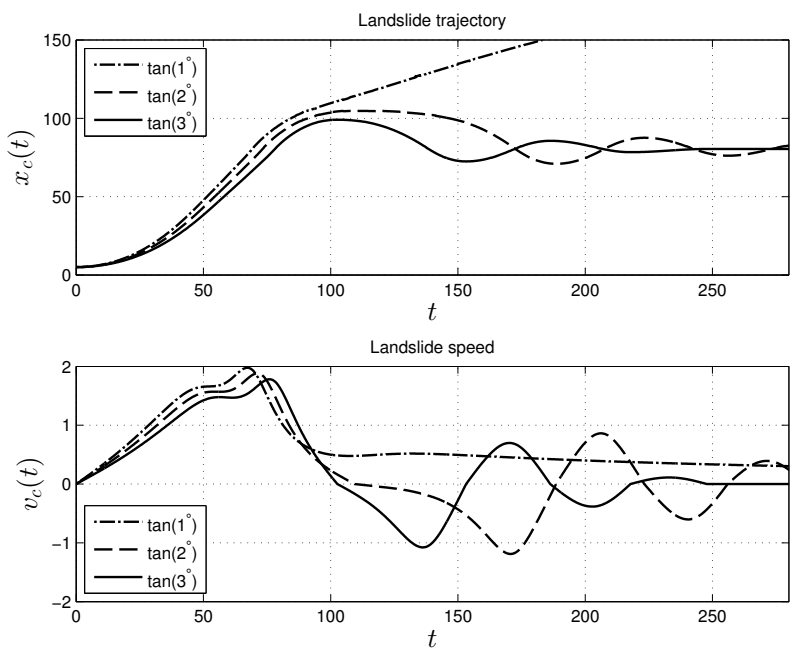

Fig. 2. Position and velocity of the barycenter of the landslide as functions of dimensional time for three different values of the friction coefficient $c_{\mathrm{f}}$.

$s(0)$ and $s^{\prime}(0)$. In the remainder we always take homogeneous initial conditions, and consider the motion driven only by the gravitational acceleration of the landslide. However, different boundary conditions might also be reasonable from a modeling point of view.

In order to approximate solutions of Eq. (4), we employ the Bogacki-Shampine third-order Runge-Kutta scheme. The integrals $\mathcal{I}_{1,2,3}(t)$ are computed using the trapezoidal rule, and once the landslide trajectory $s=s(t)$ is found, we use Eq. (2) to find its motion $x=x_{\mathrm{c}}(t)$ in the initial Cartesian coordinate system. This yields the bottom motion that drives the fluid solver.

For illustrative purposes we show a few examples of landslide trajectories over the bottom profile depicted in Fig. 1. The other parameters used in the simulations are given in Sect. 6 and also in Table 2. We performed a series of simulations in order to study the effect of various dissipative terms on the landslide trajectory. The dependence on the friction coefficient $c_{\mathrm{f}}$ is shown in Fig. 2 where the landslide barycenter position $x_{\mathrm{c}}(t)$ and its velocity $v_{\mathrm{c}}(t)$ are shown as functions of time for $c_{\mathrm{f}}=\tan \left(1^{\circ}\right), \tan \left(2^{\circ}\right)$ and $\tan \left(3^{\circ}\right)$. In the case of the weak friction $c_{\mathrm{f}}=\tan \left(1^{\circ}\right)$, the landslide reaches a sufficient speed to escape from the basin depicted in Fig. 1. For the latter case $\left(c_{\mathrm{f}}=\tan \left(3^{\circ}\right)\right)$, we show also simultaneously the landslide speed $v_{\mathrm{c}}(t)=\frac{\mathrm{d} x_{c}}{\mathrm{~d} t}$ and its acceleration $a_{\mathrm{c}}(t)=\frac{\mathrm{d} v_{\mathrm{c}}}{\mathrm{d} t}=\frac{\mathrm{d}^{2} x_{\mathrm{c}}}{\mathrm{d} t^{2}}$ in Fig. 3. In particular, one can see that the acceleration is a discontinuous function whose jumps correspond exactly to moments of time where the speed $v_{\mathrm{c}}$ changes its sign, in accordance with the employed model in Eq. (4). However, in our model there are also two new dissipative terms $F_{\mathrm{i}}$ and $F_{\mathrm{b}}$ whose importance has to be studied also. We fix the value of $c_{\mathrm{f}}=\tan \left(3^{\circ}\right)$ for all subsequent experiments, and we will vary only the two other coefficients 

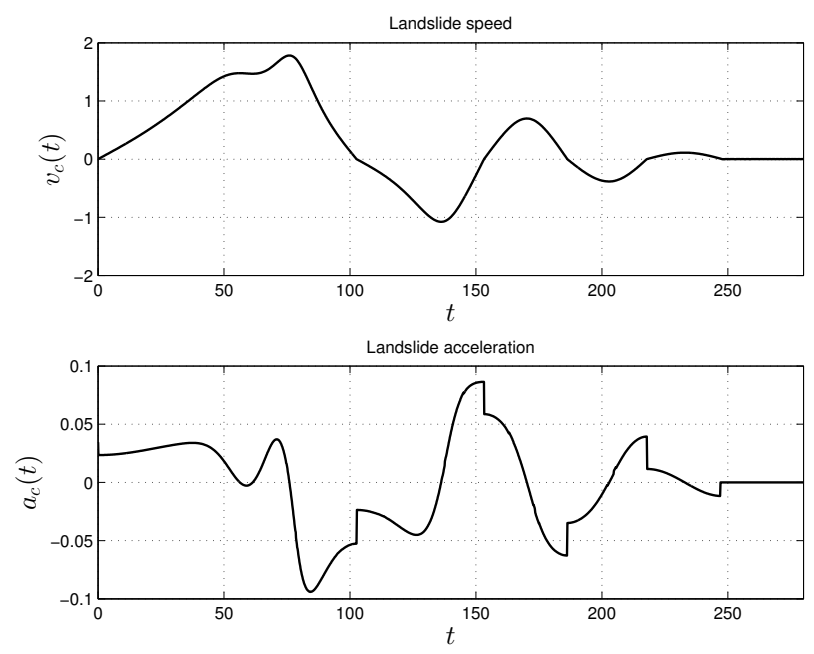

Fig. 3. Velocity and acceleration of the barycenter of the landslide as a function of dimensional time. The friction coefficient is $c_{\mathrm{f}}=\tan \left(3^{\circ}\right)$. The discontinuities in the acceleration are due to the coefficient $\operatorname{sign}\left(\frac{\mathrm{d} s}{\mathrm{~d} t}\right)$ in the definition of the friction force.
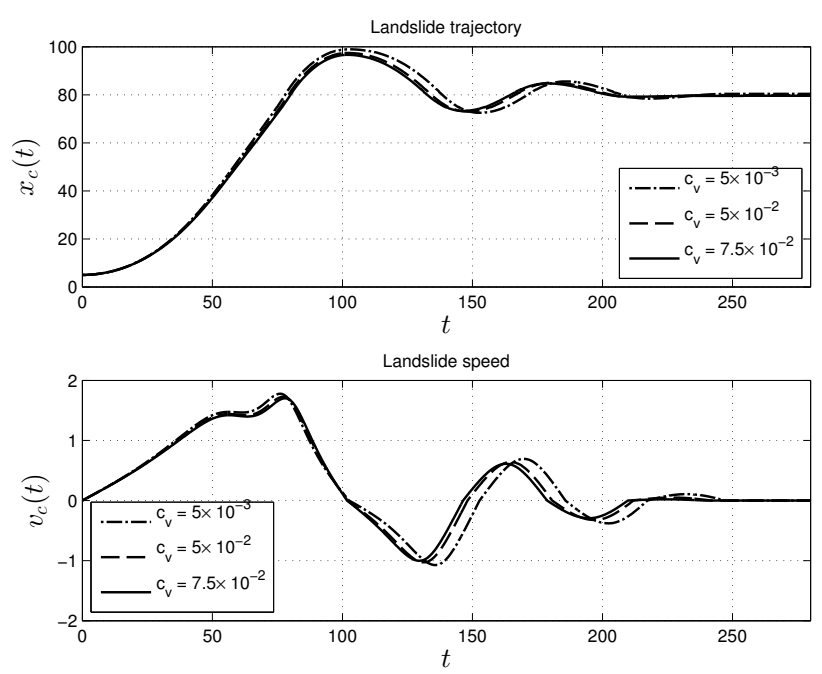

Fig. 4. Position and velocity of the barycenter of the landslide as functions of dimensional time for three different values of the friction coefficient $c_{\mathrm{v}}$.

$c_{\mathrm{v}}$ and $c_{\mathrm{b}}$ for other fixed parameters given in Table 2. These numerical results are presented in Figs. 4 and 5. One can see that the influence of these parameters on the landslide trajectory is weaker. However, we choose to keep them in the model in order to have more latitude for fine-tuning the slide trajectory if need be.

\section{The Boussinesq model}

Once the motion of the landslide is determined, and therefore the time-dependent bathymetry $h(x, t)=h_{0}(x)-\zeta(x, t)$
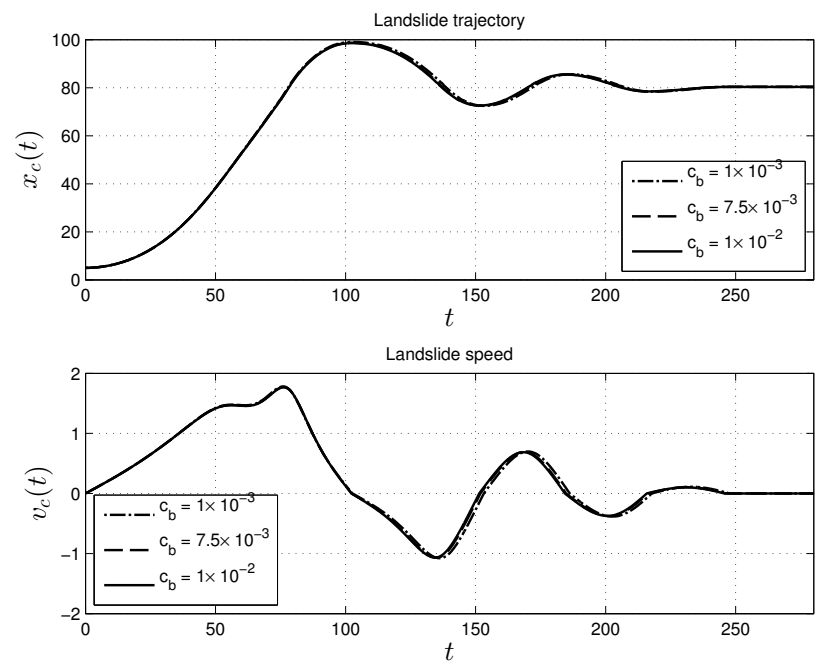

Fig. 5. Position and velocity of the barycenter of the landslide as functions of dimensional time for three different values of the friction coefficient $c_{\mathrm{b}}$.

is given, the next step is to consider the coupling between the bathymetry variations and the evolution of surface waves. The main assumptions on the fluid are that it is inviscid and incompressible, and that the flow is irrotational. Under these assumptions, the potential-flow free surface problem governs the motion of the fluid. However, in the present case, the fluid is shallow, and the waves at the surface are of small amplitude when compared to the depth of the fluid. In that case, the potential-flow problem may be simplified, and the model used in this paper is a variant of the so-called classical Boussinesq system derived by Boussinesq (1871).

Let us first consider the case of an even bottom, and a constant fluid depth $d_{0}$. Denote a typical wave amplitude by $a$, and a typical wavelength by $\lambda$. The parameter $\alpha=\frac{a}{d_{0}}$ then describes the relative amplitude of the waves, and the parameter $\beta=\frac{d_{0}^{2}}{\lambda^{2}}$ measures the "shallowness" of the fluid in comparison to the wavelength. In the case when both $\alpha$ and $\beta$ are small and approximately of the same order of magnitude, the system

$$
\begin{array}{r}
\eta_{t}+d_{0} u_{x}+(\eta u)_{x}=0, \\
u_{t}+g \eta_{x}+u u_{x}-\frac{d_{0}^{2}}{3} u_{x x t}=0
\end{array}
$$

may be used as an approximate model for the description of the evolution of the surface waves and the fluid flow. In Eq. (5), $\eta$ denotes the deflection of the free surface from its rest position, and $u$ denotes the horizontal fluid velocity at a height $z=d_{0}(-1+\sqrt{1 / 3})$ in the fluid column if $z$ is measured from the rest position of the free surface. The same equation appears if the velocity is taken to be the average of the horizontal velocity over the flow depth. 
The system in Eq. (5) was first derived by Peregrin (1967), and falls into a general class of Boussinesq systems, as shown in the systematic studies (Bona et al., 2002; Nwogu, 1993). As opposed to the shallow-water approximation, the pressure is not assumed to be hydrostatic, and the horizontal velocity varies with depth. In fact, the horizontal velocity profile is a quadratic function of $z$ (Whitham, 1974). Non-hydrostatic effects lead to the appearance of linear dispersive terms in the governing equations. The problem of landslide-generated waves has been addressed in the fully nonlinear shallow water framework (Watts et al., 2003; Chubarov et al., 2005; Yu et al., 2007; Beisel et al., 2010). Nevertheless, several authors have recently obtained interesting results even in the linear (Sammarco and Renzi, 2008; Seo and Liu, 2013) or nonlinear (Fernandez-Nieto et al., 2008; Didenkulova et al., 2010; Beisel et al., 2012) hydrostatic models.

The derivation of Eq. (5) given in Peregrin (1967) also featured an extension to non-constant but time-independent bathymetry. However, the present case of a dynamic bottom profile calls for a system that allows for time-dependent bathymetry, and such a system was derived in $\mathrm{Wu}(1987)$. Given a bottom topography described by $z=-h(x, t)$, the system takes the form

$$
\begin{array}{r}
\eta_{t}+((h+\eta) u)_{x}+h_{t}=0, \\
u_{t}+g \eta_{x}+u u_{x}=\frac{1}{2} h\left(h_{t}+(h u)_{x}\right)_{x t}-\frac{h^{2}}{6} u_{x x t} .
\end{array}
$$

In order for this system to be asymptotically valid, we need $\alpha \sim \beta$ as before. Moreover, concerning the unsteady bottom profile, we make the assumptions that $h_{x} \leq \mathcal{O}\left(\alpha \beta^{1 / 2}\right)$, and $h_{t} \leq \mathcal{O}\left(\alpha \beta^{1 / 2}\right)$.

In comparison to the shallow-water equations with a timedependent bottom topography, the system in Eq. (6) has additional terms on the right-hand side of the second equation. The effect of these terms is to incorporate frequency dispersion into the model. One practical aspect of this modification is that wave breaking can be completely avoided as long as the amplitude of the waves is small enough. Wave breaking is also possible in evolution systems of Boussinesq type (Bjørkavåg and Kalisch, 2011; Briganti et al., 2004), but the amplitudes occurring in the present problem are far from the breaking limit. The phase speed of a small-amplitude linear wave of wavelength $2 \pi / k$ in Eq. (6) with a stationary even bottom has the form $c^{2}=\frac{g d_{0}}{1+\frac{d_{0}^{2}}{3} k^{2}}$, while the phase speed is given by $c^{2}=g d_{0} \frac{\tanh \left(k d_{0}\right)}{k d_{0}}$ in the linearized full water wave problem. Thus one might argue that the dispersion in Eq. (6) is too strong in comparison with dispersion in realistic water waves. However, as discussed in Bjørkavåg and Kalisch (2011), the linear dispersion relation of Eq. (6) is still closer to the dispersion relation of the original water-wave problem than most other standard Boussinesq equations that feature even faster decay of the phase speed with increasing $k$.

\section{Solitary waves}

Before the numerical method for approximating solutions of Eq. (6) is presented, we digress for a moment, and explain how to find numerically exact solutions of the system in Eq. (5). These solutions will later be used to test the implementation of the numerical procedure. Assuming the special form

$\eta(x, t)=\eta(\xi), \quad u(x, t)=u(\xi), \quad \xi=x-c_{s} t$,

and substituting this representation into the governing Eq. (5), the following appears:

$$
\begin{aligned}
-c_{s} \eta^{\prime}+((d+\eta) u)^{\prime} & =0, \\
-c_{s} u^{\prime}+\frac{1}{2}\left(u^{2}\right)^{\prime}+g \eta^{\prime}+c_{s} \frac{d^{2}}{3} u^{\prime \prime \prime} & =0 .
\end{aligned}
$$

Assuming decay of both $\eta$ and $u$ to zero as $|x| \rightarrow \infty$, the integration of the mass conservation equation from $-\infty$ to $\xi$ gives the following relation between $\eta$ and $u$ :

$u=\frac{c_{s} \eta}{d+\eta}, \quad \eta=\frac{d \cdot u}{c_{s}-u}$.

The momentum balance equation can now be integrated to yield

$-c_{s}\left(u-\frac{d^{2}}{3} u^{\prime \prime}\right)+\frac{1}{2} u^{2}+g \eta=0$.

Finally, in order to obtain a closed form equation in terms of the velocity $u$, we substitute the expression in Eq. (7) for $\eta$ into Eq. (8). The resulting differential equation can be written in operator notation as

$\mathcal{L} u=\mathcal{N}(u)$,

where the linear operator $\mathcal{L}$ and the nonlinear operator $\mathcal{N}$ are defined respectively by

$\mathcal{L} u=c_{s}\left(u-\frac{d^{2}}{3} u^{\prime \prime}\right) \quad$ and $\mathcal{N}(u)=\frac{1}{2} u^{2}+\frac{g d u}{c_{s}-u}$.

While nothing formal appears to be known about existence of localized solutions of Eqs. (7) and (8), it is straightforward to compute approximations of solitary waves numerically. In particular, one may use the well-known Petviashvili iteration method, which takes the form

$u_{n+1}=\mathcal{L}^{-1} \cdot \mathcal{N}\left(u_{n}\right) \cdot\left(\frac{\left(u_{n}, \mathcal{N}\left(u_{n}\right)\right)}{\left(u_{n}, \mathcal{L} u_{n}\right)}\right)^{-q}$.

The exponent $q$ is usually defined as a function of the degree $p$ of the nonlinearity, with the rule of thumb that the expression $q=\frac{p}{p-1}$ generally works well. In our case, the nonlinearities are quadratic, so that we choose $p=2$, and hence $q=1$. 


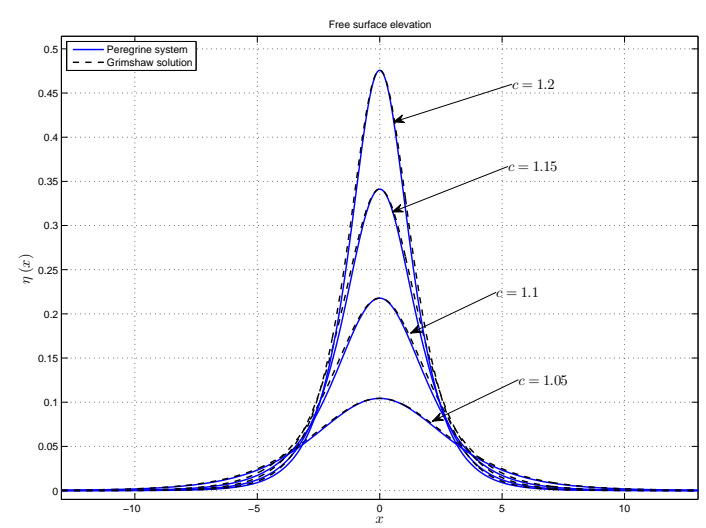

(a)

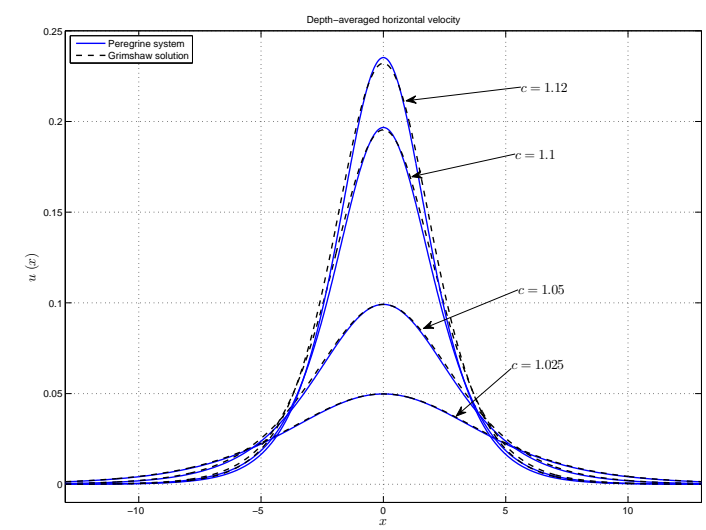

(b)

Fig. 6. Comparison of the numerical approximation of solitary wave solutions of Eq. (5) to Grimshaw's third-order asymptotic approximation of solitary waves using the Euler equations for the full water wave problem. The upper panel shows the surface elevation, and the lower panel shows the horizontal velocity at $z=d_{0}(-1+\sqrt{1 / 3})$.

The Petviashvili method was analyzed in Stepanyants and Pelinovsky (2004), and can be very efficiently implemented using the fast Fourier transform (FFT) (Frigo and Johnson, 2005). The iteration can be started for instance with the thirdorder asymptotic solution of Grimshaw (1971). The iterative procedure is continued until the $L_{\infty}$ norm between two successive iteration is on the order of machine precision. Figure 6 shows approximate solitary-wave solutions of Eq. (5) with various wave speeds, and compares them to the thirdorder asymptotic approximation of solitary-wave solutions of the full water-wave problem obtained by Grimshaw (1971). The left panel shows comparisons of the free-surface excursion, while the right panel shows a comparison of the horizontal component of the velocity field, evaluated at the non-dimensional height $\tilde{z}$ given by $\tilde{z}=-1+\sqrt{1 / 3}$. Figure 7 shows a comparison of the wave-speed-amplitude relation between the solitary-wave approximation of Eq. (5) and the ninth-order asymptotic approximation to the full water-wave problem obtained by Fenton (1972).

\section{The numerical scheme}

For the numerical discretization, a finite-volume discretization procedure similar to the one used in Barth (1994) and Barth and Ohlberger (2004) is employed. Let us take as a unit of length the undisturbed depth $d_{0}$ of the fluid above the barycenter of the landslide, and as a unit of time the ratio $\sqrt{\frac{d_{0}}{g}}$. Then the Peregrine system in Eq. (6) is rewritten in terms of the total water depth $H$ as

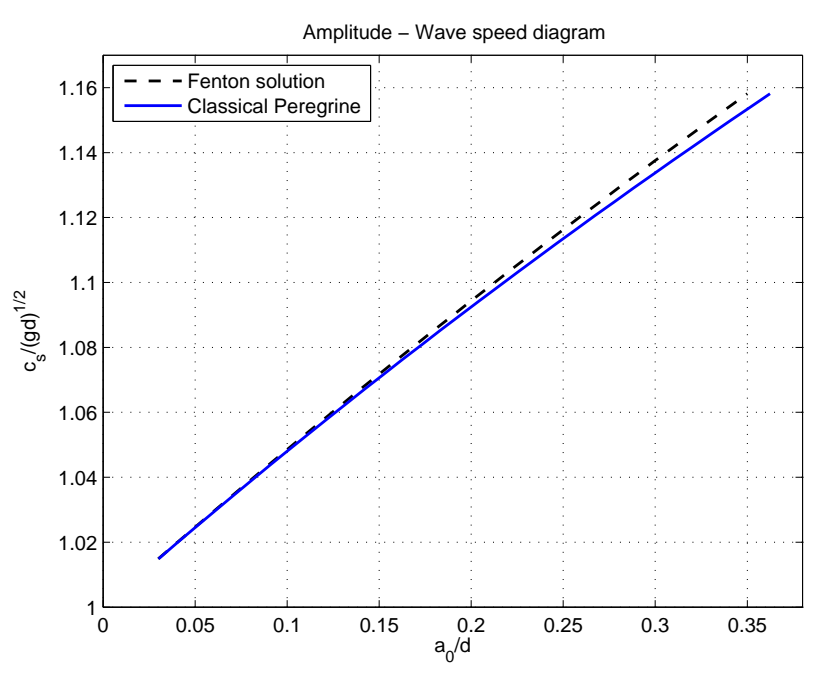

Fig. 7. Amplitude-speed relation of solitary wave solutions of Eq. (5) and of Fenton's ninth-order asymptotic approximation of solitary waves using the Euler equations for the full water wave problem.

$$
\begin{aligned}
H_{t}+[H u]_{x} & =0, \\
u_{t}+\left[\frac{1}{2} u^{2}+(H-h)\right]_{x}= & \frac{1}{2} h h_{x t t}+\frac{1}{2} h(h u)_{x x t} \\
& -\frac{1}{6} h^{2} u_{x x t} .
\end{aligned}
$$

The system in Eqs. (10) and (11) can be formally rewritten in the form

$\mathbf{V}_{t}+[\mathbb{F}(\mathbf{V})]_{x}=\mathbb{S}_{\mathrm{b}}+\mathbb{M}(\mathbf{V})$ 
where the density $\mathbf{V}$ and the advective flux $\mathbb{F}(\mathbf{V})$ are defined by

$\mathbf{V} \equiv\left(\begin{array}{l}H \\ u\end{array}\right), \quad \mathbb{F}(\mathbf{V}) \equiv\left(\begin{array}{c}H u \\ \frac{1}{2} u^{2}+(H-h)\end{array}\right)$.

The source term is defined by

$\mathbb{S}_{\mathrm{b}} \equiv\left(\begin{array}{c}0 \\ \frac{1}{2} h h_{x t t}\end{array}\right)$

and the dispersive term is defined by

$\mathbb{M}(\mathbf{V}) \equiv\left(\begin{array}{c}0 \\ \frac{1}{2} h(h u)_{x x t}-\frac{1}{6} h^{2} u_{x x t}\end{array}\right)$.

We begin our presentation with a discretization of the hyperbolic part of Eqs. (10) and (11), which is the classical nonlinear shallow-water system, and then discuss the treatment of dispersive terms. The Jacobian of the advective flux $\mathbb{F}(\mathbf{V})$ is easily computed to be

$\mathbb{A}(\mathbf{V})=\frac{\partial \mathbb{F}(\mathbf{V})}{\partial \mathbf{V}}=\left(\begin{array}{ll}u & H \\ 1 & u\end{array}\right)$,

and it is clear that $\mathbb{A}(\mathbf{V})$ has the two distinct eigenvalues

$\lambda^{ \pm}=u \pm c_{s}, \quad c_{s} \equiv \sqrt{H}$.

The corresponding right and left eigenvectors are the columns of the matrices

$R=\left(\begin{array}{cc}H & -H \\ c_{s} & c_{s}\end{array}\right), \quad L=R^{-1}=\frac{1}{2}\left(\begin{array}{cc}H^{-1} & c_{s}^{-1} \\ -H^{-1} & c_{s}^{-1}\end{array}\right)$.

We consider a partition of the real line $\mathbb{R}$ into cells (or finite volumes) $\mathcal{C}_{\mathrm{i}}=\left[x_{i-\frac{1}{2}}, x_{i+\frac{1}{2}}\right]$ with cell centers $x_{\mathrm{i}}=$ $\frac{1}{2}\left(x_{i-\frac{1}{2}}+x_{i+\frac{1}{2}}\right)(i \in \mathbb{Z})$. Let $\Delta x_{\mathrm{i}}$ denote the length of the cell $\mathcal{C}_{\mathrm{i}}$. In the following we will consider only uniform partitions with $\Delta x_{\mathrm{i}}=\Delta x, \forall i \in \mathbb{Z}$. We would like to approximate the solution $\mathbf{V}(x, t)$ by discrete values. In order to do so, we introduce the cell average of $\mathbf{V}$ on the cell $\mathcal{C}_{\mathrm{i}}$ (denoted with an overbar):

$\overline{\mathbf{V}}_{\mathrm{i}}(t) \equiv\left(\bar{H}_{\mathrm{i}}(t), \bar{u}_{\mathrm{i}}(t)\right)=\frac{1}{\Delta x} \int_{\mathcal{C}_{\mathrm{i}}} \mathbf{V}(x, t) \mathrm{d} x$.

A simple integration of Eq. (12) over the cell $\mathcal{C}_{\mathrm{i}}$ leads to the exact relation

$$
\begin{aligned}
& \frac{\mathrm{d} \overline{\mathbf{V}}}{\mathrm{d} t}+\frac{1}{\Delta x}\left[\mathbb{F}\left(\mathbf{V}\left(x_{i+\frac{1}{2}}, t\right)\right)-\right.\left.\mathbb{F}\left(\mathbf{V}\left(x_{i-\frac{1}{2}}, t\right)\right)\right] \\
&=\frac{1}{\Delta x} \int_{\mathcal{C}_{\mathrm{i}}} \mathbb{S}_{\mathrm{b}}(\mathbf{V}) \mathrm{d} x \equiv \overline{\mathbb{S}}_{\mathrm{i}}
\end{aligned}
$$

Since the discrete solution is discontinuous at cell interfaces $x_{i+\frac{1}{2}}(i \in \mathbb{Z})$, we replace the flux at the cell faces by the so-called numerical flux function

$\mathbb{F}\left(\mathbf{V}\left(x_{i \pm \frac{1}{2}}, t\right)\right) \approx \mathbb{F}_{i \pm \frac{1}{2}}\left(\overline{\mathbf{V}}_{i \pm \frac{1}{2}}^{L}, \overline{\mathbf{V}}_{i \pm \frac{1}{2}}^{R}\right)$,

where $\overline{\mathbf{V}}_{i \pm \frac{1}{2}}^{L, R}$ denotes the reconstructions of the conservative variables $\overline{\mathbf{V}}$ from left and right sides of each cell interface (the reconstruction procedure employed in the present study will be described below). Consequently, the semi-discrete scheme takes the form

$\frac{\mathrm{d} \overline{\mathbf{V}}_{\mathrm{i}}}{\mathrm{d} t}+\frac{1}{\Delta x}\left[\mathbb{F}_{i+\frac{1}{2}}-\mathbb{F}_{i-\frac{1}{2}}\right]=\overline{\mathbb{S}}_{\mathrm{i}}$

In order to discretize the advective flux $\mathbb{F}(\mathbf{V})$, we follow the method of Ghidaglia et al. $(1996,2001)$ and use the following finite volume characteristic flux (FVCF) scheme:

$\mathbb{F}(\mathbf{V}, \mathbf{W})=\frac{\mathbb{F}(\mathbf{V})+\mathbb{F}(\mathbf{W})}{2}-\mathbb{U}(\mathbf{V}, \mathbf{W}) \cdot \frac{\mathbb{F}(\mathbf{W})-\mathbb{F}(\mathbf{V})}{2}$.

The first part of the numerical flux is centered, while the second part is the upwinding introduced through the Jacobian sign-matrix $\mathbb{U}(\mathbf{V}, \mathbf{W})$ defined by

$\mathbb{U}(\mathbf{V}, \mathbf{W})=\operatorname{sign}\left[\mathbb{A}\left(\frac{1}{2}(\mathbf{V}+\mathbf{W})\right)\right]$

$\operatorname{sign}(\mathbb{A})=R \cdot \operatorname{diag}\left(s^{+}, s^{-}\right) \cdot L$,

where $s^{ \pm} \equiv \operatorname{sign}\left(\lambda^{ \pm}\right)$. After some simple algebraic computations, one can find

$\mathbb{U}=\frac{1}{2}\left(\begin{array}{cc}s^{+}+s^{-} & \left(H / c_{s}\right)\left(s^{+}-s^{-}\right) \\ \left(c_{s} / H\right)\left(s^{+}-s^{-}\right) & s^{+}+s^{-}\end{array}\right)$,

the sign-matrix $\mathbb{U}$ being evaluated at the average state of left and right values.

Finally, the source term $\mathbb{S}_{\mathrm{b}}(x, t)=\left(0, \frac{1}{2} h h_{x t t}\right)$, which is due to the moving bottom, is discretized by evaluating the bathymetry function and its derivatives at cell centers:

$\frac{1}{\Delta x} \int_{\mathcal{C}_{\mathrm{i}}} \mathbb{S}_{\mathrm{b}}(x, t) \mathrm{d} x \approx\left(0, \frac{1}{2} h\left(x_{\mathrm{i}}, t\right) h_{x t t}\left(x_{\mathrm{i}}, t\right)\right)$.

Recall that the bathymetry is composed of the static part and of the landslide subject to a translational motion:

$h(x, t)=h_{0}(x)-\zeta(x, t)=h_{0}(x)-\zeta_{0}\left(x-x_{\mathrm{c}}(t)\right)$.

The derivative $h_{x t t}$ can be readily obtained from the formula

$h_{x t t}(x, t)=\frac{\mathrm{d}^{2} x_{\mathrm{c}}}{\mathrm{d} t^{2}} \frac{\mathrm{d}^{2} \zeta_{0}}{\mathrm{~d} x^{2}}\left(x-x_{\mathrm{c}}(t)\right)-\left(\frac{\mathrm{d} x_{\mathrm{c}}}{\mathrm{d} t}\right)^{2} \frac{\mathrm{d}^{3} \zeta_{0}}{\mathrm{~d} x^{3}}\left(x-x_{\mathrm{c}}(t)\right)$. 


\subsection{High-order reconstruction}

In order to obtain a higher order scheme in space, we need to replace the piecewise constant data by a piecewise polynomial representation. This goal is achieved by various socalled reconstruction procedures such as MUSCL TVD (Kolgan, 1975; van Leer, 1979, 2006), UNO (Harten and Osher, 1987), ENO (Harten, 1989), WENO (Xing and Shu, 2005) and many others. In recent studies on unidirectional wave models (Dutykh et al., 2013a) and on Boussinesq-type equations (Dutykh et al., 2011b), the UNO2 scheme showed a good performance with small dissipation in realistic propagation and run-up simulations. Consequently, we retain this scheme for the discretization of the advective flux of the Peregrine system in Eqs. (10) and (11).

The main idea of the UNO2 scheme is to construct a nonoscillatory piecewise-parabolic interpolant $\mathbf{Q}(x)$ to a piecewise smooth function $\mathbf{V}(x)$ (see Harten and Osher, 1987, for more details). On each segment containing the face $x_{i+\frac{1}{2}} \in$ $\left[x_{\mathrm{i}}, x_{i+1}\right]$, the function $\mathbf{Q}(x)=\mathbf{q}_{i+\frac{1}{2}}(x)$ is locally a quadratic polynomial and wherever $v(x)$ is smooth we have

$$
\begin{aligned}
\mathbf{Q}(x)-\mathbf{V}(x) & =\mathbf{0}+\mathcal{O}\left(\Delta x^{3}\right), \\
\frac{\mathrm{d} \mathbf{Q}}{\mathrm{d} x}(x \pm 0)-\frac{\mathrm{d} \mathbf{V}}{\mathrm{d} x} & =\mathbf{0}+\mathcal{O}\left(\Delta x^{2}\right) .
\end{aligned}
$$

Also, $\mathbf{Q}(x)$ should be non-oscillatory in the sense that the number of its local extrema does not exceed that of $\mathbf{V}(x)$. Since $\mathbf{q}_{i+\frac{1}{2}}\left(x_{\mathrm{i}}\right)=\overline{\mathbf{V}}_{\mathrm{i}}$ and $\mathbf{q}_{i+\frac{1}{2}}\left(x_{i+1}\right)=\overline{\mathbf{V}}_{i+1}$, it can be written in the form

$$
\begin{aligned}
& \mathbf{q}_{i+\frac{1}{2}}(x)=\overline{\mathbf{V}}_{\mathrm{i}}+ \\
& \mathfrak{d}_{i+\frac{1}{2}}\{\mathbf{V}\} \times \frac{x-x_{\mathrm{i}}}{\Delta x}+\frac{1}{2} \mathfrak{D}_{i+\frac{1}{2}}\{\mathbf{V}\} \times \frac{\left(x-x_{\mathrm{i}}\right)\left(x-x_{i+1}\right)}{\Delta x^{2}},
\end{aligned}
$$

where $\mathfrak{d}_{i+\frac{1}{2}}\{\mathbf{V}\} \equiv \overline{\mathbf{V}}_{i+1}-\overline{\mathbf{V}}_{i}$ and $\mathfrak{D}_{i+\frac{1}{2}} \mathbf{V}$ is closely related to the second derivative of the interpolant since $\mathfrak{D}_{i+\frac{1}{2}}\{\mathbf{V}\}=$ $\Delta x^{2} \mathbf{q}_{i+\frac{1}{2}}^{\prime \prime}(x)$. The polynomial $\mathbf{q}_{i+\frac{1}{2}}(x)$ is chosen to be the least oscillatory between two candidates interpolating $\mathbf{V}(x)$ at $\left(x_{i-1}, x_{\mathrm{i}}, x_{i+1}\right)$ and $\left(x_{\mathrm{i}}, x_{i+1}, x_{i+2}\right)$. This requirement leads to the following choice of $\mathfrak{D}_{i+\frac{1}{2}}\{\mathbf{V}\} \equiv$ $\operatorname{minmod}\left(\mathfrak{D}_{\mathrm{i}}\{\mathbf{V}\}, \mathfrak{D}_{i+1}\{\mathbf{V}\}\right)$ with

$$
\begin{aligned}
\mathfrak{D}_{\mathrm{i}}\{\mathbf{V}\} & =\overline{\mathbf{V}}_{i+1}-2 \overline{\mathbf{V}}_{\mathrm{i}}+\overline{\mathbf{V}}_{i-1}, \\
\mathfrak{D}_{i+1}\{\mathbf{V}\} & =\overline{\mathbf{V}}_{i+2}-2 \overline{\mathbf{V}}_{i+1}+\overline{\mathbf{V}}_{\mathrm{i}},
\end{aligned}
$$

and where minmod $(x, y)$ is the usual minmod function defined as

$\operatorname{minmod}(x, y) \equiv \frac{1}{2}[\operatorname{sign}(x)+\operatorname{sign}(y)] \times \min (|x|,|y|)$.

To achieve the second order $\mathcal{O}\left(\Delta x^{2}\right)$ accuracy, it is sufficient to consider piecewise linear reconstructions in each cell. Let $L(x)$ denote this approximately reconstructed function, which can be written in the form

$L(x)=\overline{\mathbf{V}}_{\mathrm{i}}+\mathbf{S}_{\mathrm{i}} \cdot \frac{x-x_{\mathrm{i}}}{\Delta x}, \quad x \in\left[x_{i-\frac{1}{2}}, x_{i+\frac{1}{2}}\right]$.

In order for $L(x)$ to be a non-oscillatory approximation, we use the parabolic interpolation $\mathbf{Q}(x)$ constructed below to estimate the slopes $\mathbf{S}_{\mathrm{i}}$ within each cell:

$\mathbf{S}_{\mathrm{i}}=\Delta x \times \operatorname{minmod}\left(\frac{\mathrm{d} \mathbf{Q}}{\mathrm{d} x}\left(x_{\mathrm{i}}-0\right), \frac{\mathrm{d} \mathbf{Q}}{\mathrm{d} x}\left(x_{\mathrm{i}}+0\right)\right)$.

In other words, the solution is reconstructed on the cells while the solution gradient is estimated on the dual mesh as it is often performed in more modern schemes (Barth, 1994; Barth and Ohlberger, 2004). A brief summary of the UNO2 reconstruction can be also found in Dutykh et al. (2011b, 2013a).

\subsection{Treatment of the dispersive terms}

In this section, we explain how to treat the relevant dispersive terms in the second Eq. (11) of the Peregrine system numerically. We propose the following approximation for the second component of $M(\overline{\mathbf{V}})$ of $\mathbb{M}(\overline{\mathbf{V}})$ :

$$
\begin{aligned}
M_{\mathrm{i}}(\overline{\mathbf{V}})= & \frac{1}{2} \bar{h}_{\mathrm{i}} \frac{\bar{h}_{i+1}\left(\bar{u}_{t}\right)_{i+1}-2 \bar{h}_{i}\left(\bar{u}_{t}\right)_{i}+\bar{h}_{i-1}\left(\bar{u}_{t}\right)_{i-1}}{\Delta x^{2}} \\
& -\frac{1}{6} \bar{h}_{\mathrm{i}}^{2} \frac{\left(\bar{u}_{t}\right)_{i+1}-2\left(\bar{u}_{t}\right)_{i}+\left(\bar{u}_{t}\right)_{i-1}}{\Delta x^{2}} \\
= & \frac{\bar{h}_{\mathrm{i}}}{2 \Delta x^{2}}\left(\bar{h}_{i-1}-\frac{1}{3} \bar{h}_{\mathrm{i}}\right)\left(\bar{u}_{t}\right)_{i-1} \\
& -\frac{2}{3 \Delta x^{2}} \bar{h}_{\mathrm{i}}^{2}\left(\bar{u}_{t}\right)_{i}+\frac{\bar{h}_{\mathrm{i}}}{2 \Delta x^{2}}\left(\bar{h}_{i+1}-\frac{1}{3} \bar{h}_{\mathrm{i}}\right)\left(\bar{u}_{t}\right)_{i+1} .
\end{aligned}
$$

Note that this spatial discretization is of the second order $\mathcal{O}\left(\Delta x^{2}\right)$ so as to be consistent with the UNO2 advective flux discretization presented above. If we denote by $I$ the identity matrix, we can now rewrite the semi-discrete scheme in the form

$$
\begin{aligned}
\frac{\mathrm{d} \bar{H}}{\mathrm{~d} t}+\frac{1}{\Delta x}\left[\mathbb{F}_{+}^{(1)}(\overline{\mathbf{V}})-\mathbb{F}_{-}^{(1)}(\overline{\mathbf{V}})\right] & =0, \\
(I-M) \cdot \frac{\mathrm{d} \bar{u}}{\mathrm{~d} t}+\frac{1}{\Delta x}\left[\mathbb{F}_{+}^{(2)}(\overline{\mathbf{V}})-\mathbb{F}_{-}^{(2)}(\overline{\mathbf{V}})\right] & =\mathbb{S}_{\mathrm{b}}^{(2)},
\end{aligned}
$$

where $\mathbb{F}_{ \pm}^{(1,2)}(\overline{\mathbf{V}})$ are the two components of the advective numerical flux vector $\mathbb{F}$ at the right $(+)$ and left $(-)$ faces correspondingly, and $\mathbb{S}_{\mathrm{b}}^{(2)}$ denotes the discretization of the second component of $\mathbb{S}_{\mathrm{b}}$.

In order to advance the numerical solution forward in time, one has to invert the matrix $(I-M)$ at every time step. This is no problem in practice, since the matrix appears to be well conditioned in all cases we have considered. In fact, the invertibility of the matrix $(I-M)$ can be rigorously shown to hold for small enough $\Delta x$ since the matrix is then diagonally dominant. The criterion for diagonal dominance in the 
present case is seen to be

$$
\begin{aligned}
1+\frac{2}{3 \Delta x^{2}} \bar{h}_{\mathrm{i}}^{2}>\left|-\frac{1}{6} \frac{\bar{h}_{\mathrm{i}}^{2}}{\Delta x^{2}}+\frac{\bar{h}_{\mathrm{i}}}{2 \Delta x^{2}} \bar{h}_{i-1}\right|+ \\
\left|-\frac{1}{6} \frac{\bar{h}_{\mathrm{i}}^{2}}{\Delta x^{2}}+\frac{\bar{h}_{\mathrm{i}}}{2 \Delta x^{2}} \bar{h}_{i+1}\right| .
\end{aligned}
$$

Using a Taylor expansion to express the terms $\bar{h}_{i-1}$ and $\bar{h}_{i+1}$ as $\bar{h}_{i-1}=\bar{h}_{\mathrm{i}}-\Delta x \bar{h}^{\prime}\left(x_{\mathrm{i}}\right)+\mathcal{O}\left(\Delta x^{2}\right)$ and $\bar{h}_{i+1}=\bar{h}_{\mathrm{i}}+$ $\Delta x \bar{h}^{\prime}\left(x_{\mathrm{i}}\right)+\mathcal{O}\left(\Delta x^{2}\right)$, respectively, the criterion reduces to $1+\frac{1}{3 \Delta x^{2}} \bar{h}_{\mathrm{i}}^{2}>\frac{\bar{h}_{\mathrm{i}} \bar{h}_{\mathrm{i}}^{\prime}}{\Delta x}+\mathcal{O}(1)$, and this is guaranteed to hold for small enough $\Delta x$.

\subsection{Time stepping}

We assume that the linear system of equations is already inverted, and we have the following system of ordinary differential equations:

$\mathbf{V}_{t}=\mathcal{N}(\mathbf{V}, t), \quad \mathbf{V}(0)=\mathbf{V}_{0}$.

In order to solve numerically the last system of equations, we apply the Bogacki-Shampine method proposed by Przemyslaw Bogacki and Lawrence F. Shampine in 1989 (Bogacki and Shampine, 1989). It is a Runge-Kutta scheme of the third order with four stages. It has an embedded secondorder method that is used to estimate the local error and thus, to adapt the time step size. Moreover, the Bogacki-Shampine method enjoys the first same as last (FSAL) property so that it needs approximately three function evaluations per step. This method is also implemented in the ode23 function in MATLAB (Shampine and Reichelt, 1997). The one step of the Bogacki-Shampine method is given by

$$
\begin{aligned}
k_{1} & =\mathcal{N}\left(\mathbf{V}^{(n)}, t_{n}\right), \\
k_{2} & =\mathcal{N}\left(\mathbf{V}^{(n)}+\frac{1}{2} \Delta t_{n} k_{1}, t_{n}+\frac{1}{2} \Delta t\right), \\
k_{3} & \left.=\mathcal{N}\left(\mathbf{V}^{(n)}\right)+\frac{3}{4} \Delta t_{n} k_{2}, t_{n}+\frac{3}{4} \Delta t\right), \\
\mathbf{V}^{(n+1)} & =\mathbf{V}^{(n)}+\Delta t_{n}\left(\frac{2}{9} k_{1}+\frac{1}{3} k_{2}+\frac{4}{9} k_{3}\right), \\
k_{4} & =\mathcal{N}\left(\mathbf{V}^{(n+1)}, t_{n}+\Delta t_{n}\right), \\
\mathbf{V}_{2}^{(n+1)} & =\mathbf{V}^{(n)}+\Delta t_{n}\left(\frac{4}{24} k_{1}+\frac{1}{4} k_{2}+\frac{1}{3} k_{3}+\frac{1}{8} k_{4}\right) .
\end{aligned}
$$

Here $\mathbf{V}^{(n)} \approx \mathbf{V}\left(t_{n}\right), \Delta t$ is the time step and $\mathbf{V}_{2}^{(n+1)}$ is a second-order approximation to the solution $\mathbf{V}\left(t_{n+1}\right)$, so the difference between $\mathbf{V}^{(n+1)}$ and $\mathbf{V}_{2}^{(n+1)}$ gives an estimation of the local error. The FSAL property consists in the fact that $k_{4}$ is equal to $k_{1}$ in the next time step, thus saving one function evaluation.

If the new time step $\Delta t_{n+1}$ is given by $\Delta t_{n+1}=$ $\rho_{n} \Delta t_{n}$, then, according to the $\mathrm{H} 211 \mathrm{~b}$ digital filter approach (Söderlind, 2003; Söderlind and Wang, 2006), the proportionality factor $\rho_{n}$ is given by

$\rho_{n}=\left(\frac{\delta}{\epsilon_{n}}\right)^{\beta_{1}}\left(\frac{\delta}{\epsilon_{n-1}}\right)^{\beta_{2}} \rho_{n-1}^{-\alpha}$, where $\epsilon_{n}$ is a local error estimation at time step $t_{n}$, and the constants $\beta_{1}, \beta_{2}$ and $\alpha$ are defined by

$\alpha=\frac{1}{4}, \quad \beta_{1}=\frac{1}{4 p}, \quad \beta_{2}=\frac{1}{4 p}$.

The parameter $p$ gives the order of the scheme, and $p=3$ in our case.

The adaptive strategy in Eq. (14) can be further improved if we smooth the factor $\rho_{n}$ before computing the next time step $\Delta t_{n+1}$ :

$\Delta t_{n+1}=\hat{\rho}_{n} \Delta t_{n}, \quad \hat{\rho}_{n}=\omega\left(\rho_{n}\right)$.

The function $\omega(\rho)$ is called the time step limiter and should be smooth, monotonically increasing and should satisfy the following conditions:

$\omega(0)<1, \quad \omega(+\infty)>1, \quad \omega(1)=1, \omega^{\prime}(1)=1$.

One possible choice was suggested in Söderlind and Wang (2006):

$\omega(\rho)=1+\kappa \arctan \left(\frac{\rho-1}{\kappa}\right)$.

In our computations the parameter $\kappa$ is set to 1 .

\subsection{Validation}

The scheme described in this section is implemented in MAT$\mathrm{LAB}$, and runs on a workstation. To check whether the implementation is correct, we use the approximate solitary waves of Eq. (5), computed in the last section. These are used as initial data in the fully discrete scheme, and integrated forward in time. The computed solutions are then compared to the same solitary waves, but shifted forward in space by $c t_{0}$, where $c$ is the wave speed, and $t_{0}$ is the final time. This procedure is repeated a number of times with different spatial grid sizes. As a result, it is possible to find the spatial convergence rate of the scheme. As is visible in Fig. 8, the convergence achieved by the practical implementation of the discretization is very close to the theoretical convergence rate. Since the temporal discretization is adaptive, we do not present a convergence study in terms of the timestep $\Delta t$.

\subsubsection{Wave generation by moving bottom}

We have just shown the convergence of our scheme under the mesh refinement. Even if the solution we used in validation is fully nonlinear, it only exists on the flat bottom. Since in the present study we are mainly interested in the wave generation by bottom motion, the next validation test will be entirely devoted to this question. Namely, we are going to use an analytical solution to the linearized full Euler equations also known as the Cauchy-Poisson problem. The use of this solution in tsunami generation problems was first proposed by Hammack (1973). 


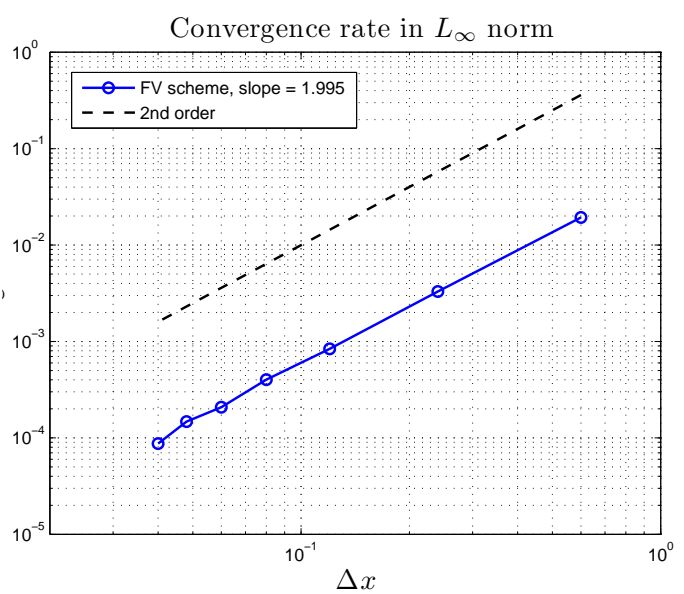

(a)

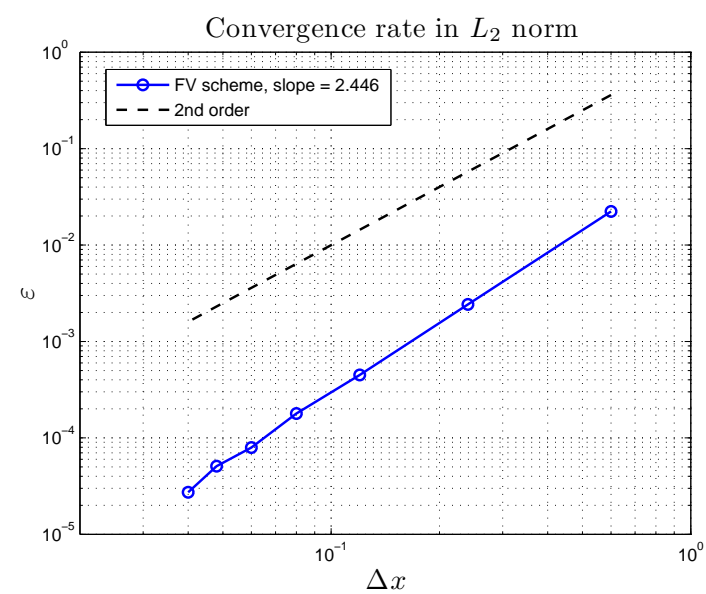

(b)

Fig. 8. Convergence rate of the finite-volume scheme in the $L^{\infty}$-norm (left panel) and the $L^{2}$-norm (right panel). The numerical integration of a solitary wave as shown in Fig. 6 is compared to a translated profile. It appears that the second-order convergence is achieved.

We consider the linearized water wave problem for a fluid layer of uniform depth $z=-d_{0}=$ const. However, a portion of the bottom can move vertically, and the bottom deformation is given by a smooth function $\zeta(x, t)$, such that $\zeta(x, 0)=0$. At any given time $t$, the bottom profile is given by $z=-d_{0}+\zeta(x, t)$. Moreover, we will make a special assumption about the structure of the bottom deformation:

$\zeta(x, t)=T(t) \zeta_{0}(x), \quad T(t)=1-\mathrm{e}^{-\alpha t}, \quad \alpha>0, \quad t \geq 0$.

Obviously, we have to assume that $\left\|\zeta_{0}\right\| \ll 1$ so that the linear approximation be valid. Then, the free surface elevation at any time is given by the following formula (Hammack, 1973; Dutykh et al., 2006):

$$
\begin{aligned}
\eta(x, t)=-\frac{\alpha^{2}}{2 \pi} \int_{\mathbb{R}} \frac{\hat{\zeta}_{0}(k)}{\cosh \left(k d_{0}\right)} \times \\
\quad \frac{\mathrm{e}^{-\alpha t}-\cos (\omega t)-\frac{\omega}{\alpha} \sin (\omega t)}{\alpha^{2}+\omega^{2}} \cdot \mathrm{e}^{-\mathrm{i} k x} \mathrm{~d} k,
\end{aligned}
$$

where $\hat{\zeta}_{0}(k)$ is the Fourier transform of $\zeta_{0}(x)$ and $\omega=$ $\sqrt{g k \tanh \left(k d_{0}\right)}$ is the wave frequency corresponding to the wavenumber $k$. The above integral can be easily computed using the FFT algorithm. To fix the ideas for numerical computations, we will take the following localized oscillatory bottom deformation:

$\zeta_{0}(x)=a \cos \left(k_{0} x\right) \mathrm{e}^{-\lambda_{0} x^{2}}, \quad \lambda_{0}>0$.

The values of all parameters used in numerical simulation are given in Table 1 . The nonlinearity parameter $a / d_{0}$ is chosen to be 0.05 , which is far above the nonlinearity of the earthquake-generated tsunamis. However, we think that this value corresponds better to the scope of the present
Table 1. Values of various parameters used to simulate the wave generation by moving bottom.

\begin{tabular}{lc}
\hline Gravity acceleration: $g$ & 1.0 \\
Gravity acceleration: $g$ & 1.0 \\
Undisturbed water depth: $d_{0}$ & 1.0 \\
Bottom displacement amplitude: $a$ & 0.05 \\
Bottom oscillation inverse length: $k_{0}$ & $\frac{\pi}{40}$ \\
Bottom localization parameter: $\lambda_{0}$ & $0.7 \times 10^{-3}$ \\
Vertical uplift speed: $\alpha$ & 1.0 and 2.0 \\
\hline
\end{tabular}

study. In order to simulate this setup using the Peregrine system, we consider a symmetric 1-D computational domain [-220,220] discretized into $N=2000$ equal control volumes. The time stepping tolerance parameter was set far below the spatial discretization error $\left(\sim \mathcal{O}\left(\Delta x^{2}\right)\right)$. First, we will take a moderately fast bottom uplift corresponding to the parameter $\alpha=1.0$. Computational results are presented in Fig. 9a-e. One can see that the overall agreement is fairly good even if some small differences can be noticed in Fig. 9c-d. However, the resulting wave form predicted by the Peregrine system follows closely the linearized full Euler solution in Eq. (15). Now, we will double the bottom uplift speed $(\alpha=2.0)$. This result is presented in Fig. 10a-e. One can see more substantial differences during the generation phase (see panels b-d). However, here again the resulting wave is surprisingly well represented by the Boussinesq-type equations. The observed discrepancies during the generation phase are essentially due to the simplified structure of the vertical speed in Boussinesq-type equations (Dutykh et al., 2013b). 


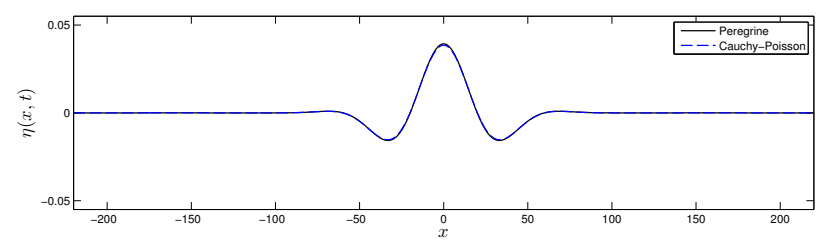

(a) $t=1.5$

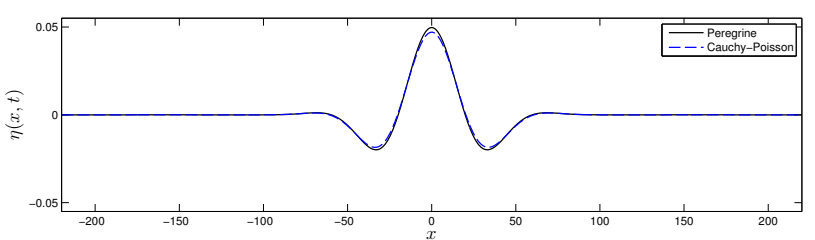

(b) $t=4.0$

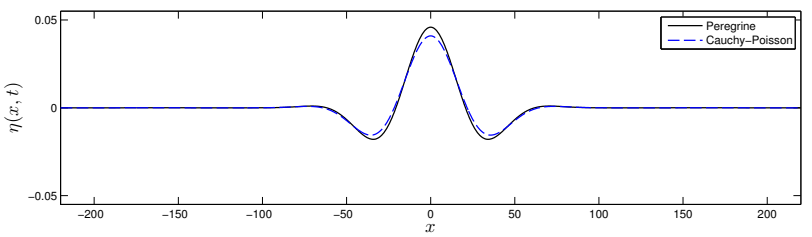

(c) $t=8.0$

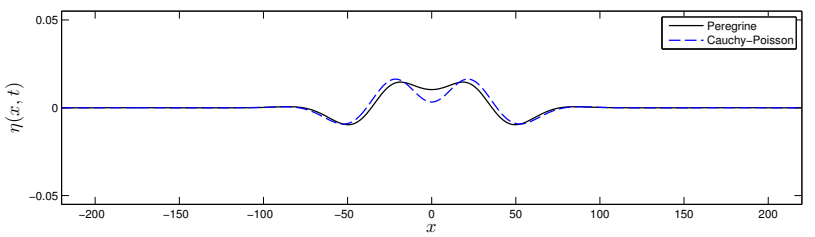

(d) $t=20.0$

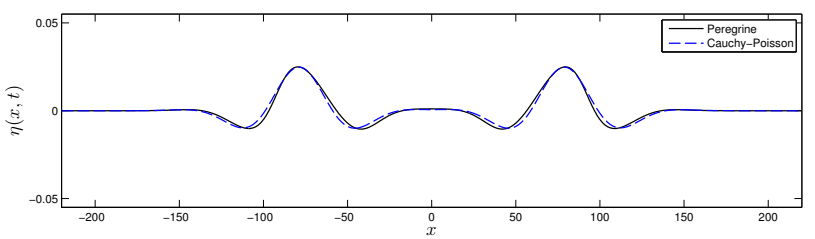

(e) $t=80.0$

Fig. 9. Free surface waves generated by a moderately fast bottom motion. The blue dashed line corresponds to the analytical CauchyPoisson solution, while the solid black line is our numerical solution to the Peregrine system. The time snapshots are taken at $t=1.5$, $t=4, t=8, t=20$, and $t=80$, from top to bottom.

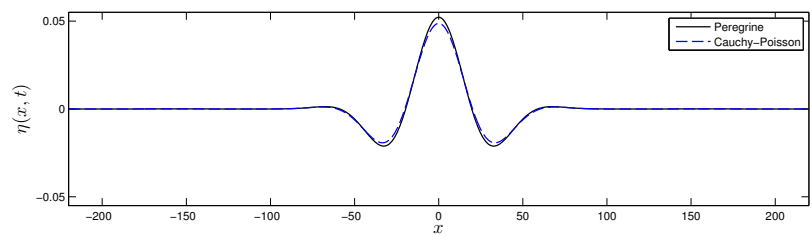

(a) $t=2.5$

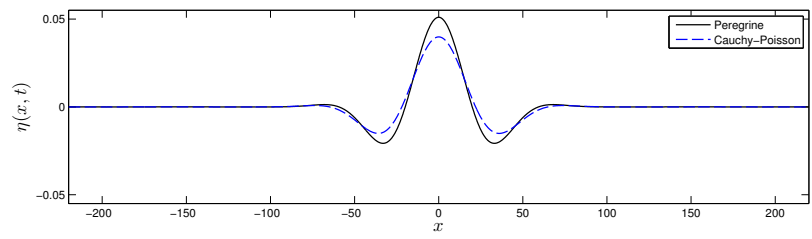

(b) $t=8.0$

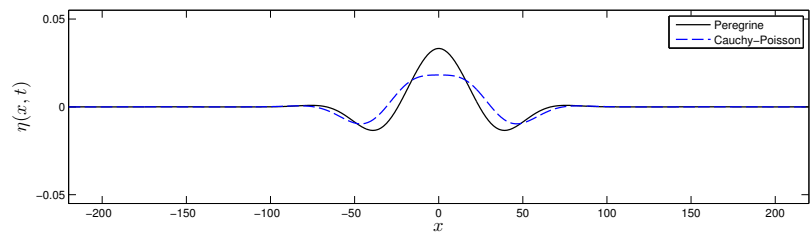

(c) $t=15.0$

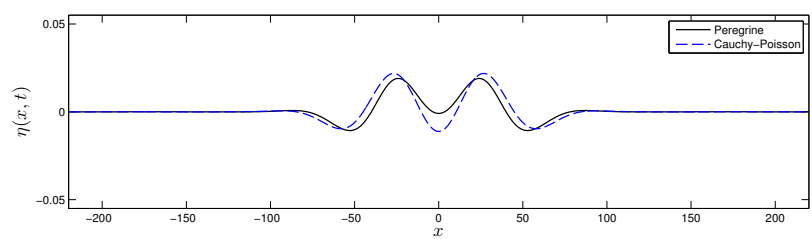

(d) $t=25.0$

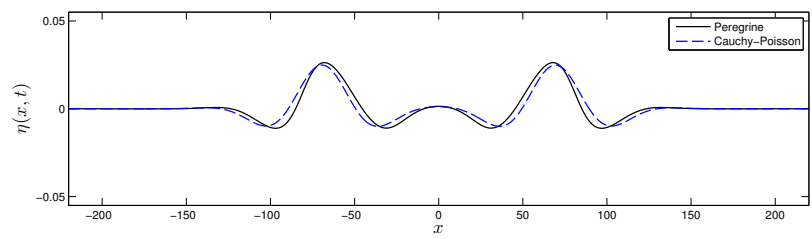

(e) $t=70.0$

Fig. 10. Free surface waves generated by a fast bottom motion. The blue dashed line corresponds to the analytical Cauchy-Poisson solution, while the solid black line is our numerical solution to the Peregrine system. The time snapshots are taken at $t=2.5, t=8$, $t=15, t=25$, and $t=70$, from top to bottom. 


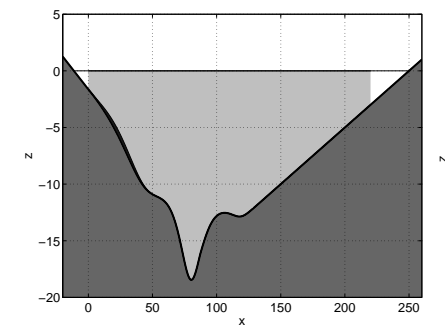

(a)

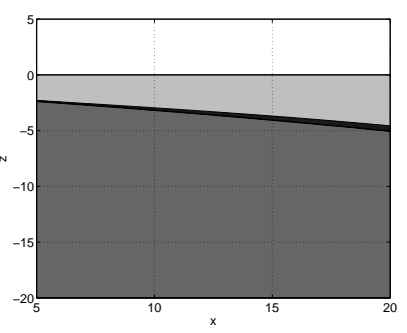

(b)
Fig. 11. The physical setup of the problem. The riverbed is indicated in dark grey. The computational fluid domain is shaded light grey, and the landslide is visible in black. Note the difference in horizontal and vertical scales in the left panel. The upper panel shows a close-up of the left beach and part of the landslide in a one-to-one aspect ratio.

\section{Numerical results}

Let us consider a one-dimensional computational domain $I=[a, b]=[0,220]$ composed of two regions: the generation region and a sloping beach on the right. More specifically, the static bathymetry function $h_{0}(x)$ is given by a smoothed out profile generated from the expression

$h_{0}(x)= \begin{cases}d_{0}+\tan \delta(x-a)+p(x), & a \leq x \leq m, \\ d_{0}+\tan \delta(m-a)-\tan \delta(x-m), & x>m,\end{cases}$

where the function $p(x)$ is defined as

$p(x)=A_{1} \operatorname{sech}\left(k_{1}\left(x-x_{1}\right)\right)+A_{2} \operatorname{sech}\left(k_{2}\left(x-x_{2}\right)\right)$.

In essence, this function represents a perturbation of the sloping bottom by two underwater bumps. We made this nontrivial choice in order to illustrate the advantages of our landslide model, which was designed to handle general nonflat bathymetries. The parameters can be chosen in order to fit a given bathymetry, but the particular values used here are $A_{1}=4.75, A_{2}=8.85, k_{1}=0.06, k_{2}=0.13, x_{1}=45$, $x_{2}=80$, and $m=120$. The bottom profile for these parameters is depicted in Fig. 11. Of course, in general, if the bottom topography is known, then a numerical bathymetry map could also be used.

We now present some results of the solution of the surface wave problem using the model in Sect. 3 integrated numerically with the method of Sect. 5. A landslide is introduced on the left side of the bathymetry, and using the method of Sect. 2, its path along the bottom is determined by following the barycenter. Simultaneously, the system in Eq. (6) is solved with the time-dependent bottom topography given from the solution of the landslide problem. The problem is integrated up to a final time $T$. Figure 12 shows wave records at six virtual wave gauges for both the dispersive system in Eq. (6) and the shallow-water system. It appears from this figure that the shallow-water system underpredicts the development of free-surface oscillations. In particular, the wave gauges located at $x=40$ and $x=60$ show similar wave heights for both the shallow-water and the dispersive system, but a qualitative divergence, as small oscillations are already developing that are not captured by the shallow-water system. Once the waves have propagated to the wave gauges located at $x=80$, the dispersive oscillations have amplified, so that the wave height is larger by a factor of 2 to 3 than the wave height predicted by the shallow-water system. Going further to the wave gauges located at $x=100$ and $x=120$, the now rising bottom starts to have a damping effect on the waves.

The maximum and minimum free surface elevation over the whole domain are shown in Fig. 13. On the lower panel of the same Fig. 13, we show the maximal unsigned horizontal velocity. One can see that for short times the hydrostatic and dispersive models give very close extreme values. Later the differences start to appear due to the accumulation of dispersive effects.

Figure 14 shows the development of the kinetic energy of the landslide mass and simultaneously the total (kinetic plus potential) energy contained in the body of the fluid and the surface waves. Energy development is an important question in the study of tsunamis, and there have been studies exclusively devoted to this question (Tinti and Bortolucci, 2000). Energy issues connected to water wave models of Boussinesq-type have also been studied before (Ali and Kalisch, 2010, 2012; Dutykh and Dias, 2009). While these models contained a source of energy, in the case at hand, the work done by friction as the landslide slides down the bottom acts as a drain of energy, and after the landslide has come to rest, all energy has been transferred to the fluid. However, not all energy can be considered as residing in the wave motion, because a significant amount of energy is needed to lift the water from the final position of the landslide to the initial position of the landslide. This results in a large increase in potential energy of the fluid, and only a fraction of the potential energy of the landslide is transferred to the wave motion. This fact has also been explained in previous works (Harbitz et al., 2006).

In order to compute the wave energy in the fluid, we use the integral

$E_{\mathrm{w}}=\int_{a}^{b}\left\{\frac{g}{2} \eta^{2}+\frac{1}{2}\left(h_{0}+\eta\right) u^{2}\right\} \mathrm{d} x$,

which arises from the shallow-water theory. The kinetic energy of the landslide is given by

$E_{s l}=\frac{1}{2} m v^{2}$,

with the generalized mass $m$ given by Eq. (3), and $v=\frac{\mathrm{d} s}{\mathrm{~d} t}$ as defined in Sect. 2. Figure 14 shows the development of the wave energy and kinetic energy of the landslide. The upper panel shows the energy according to the shallow-water and 
Table 2. Values of various parameters used in numerical computations.

\begin{tabular}{llrr}
\hline Symbol & Parameter & Units & Values \\
\hline$g$ & gravitational acceleration & $\mathrm{m} \mathrm{s}^{-2}$ & 9.81 \\
$d_{0}$ & water depth at $x=a$ & $\mathrm{~m}$ & $1.0-2.0$ \\
$\tan (\delta)$ & bottom slope & & 0.1 \\
$A$ & landslide amplitude & $\mathrm{m}$ & 0.55 \\
$l$ & landslide length & $\mathrm{m}$ & 52.4 \\
$c_{\mathrm{W}}$ & added mass coefficient & & 1.0 \\
$c_{\mathrm{d}}$ & water drag coefficient & & 1.0 \\
$c_{\mathrm{f}}$ & friction coefficient & & $\tan \left(3^{\circ}\right)$ \\
$\gamma$ & density ratio water/landslide & 1.8 \\
$c_{\mathrm{b}}$ & friction coefficient with bottom & & $7.63 \times 10^{-4}$ \\
$c_{\mathrm{V}}$ & viscous friction coefficient & & $1.27 \times 10^{-3}$ \\
\hline
\end{tabular}
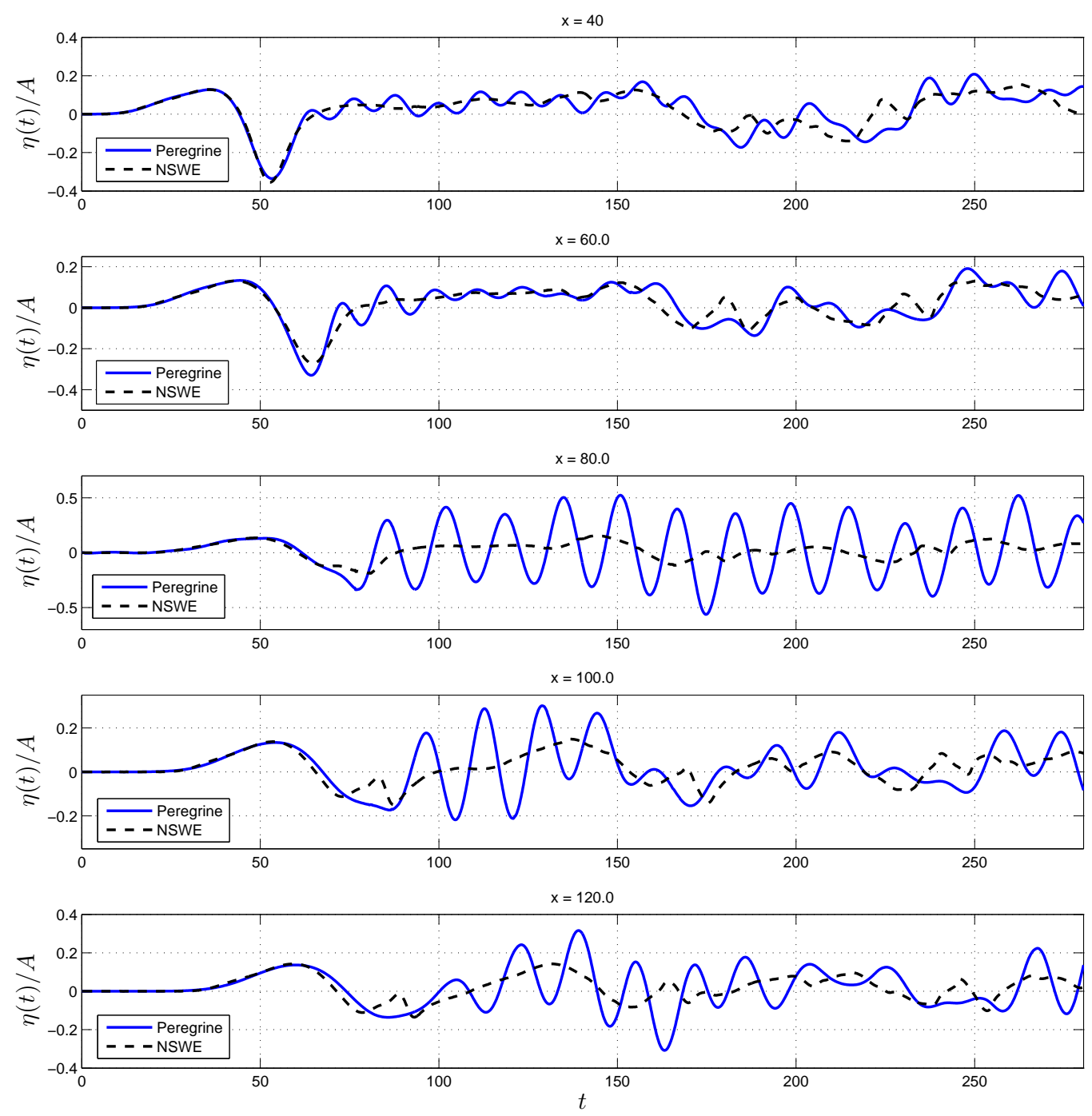

Fig. 12. Time series of the surface elevation at wave gauges located at $x=40, x=60, x=80, x=100$ and $x=120$. The solid (blue) curve depicts the wave elevation computed with the dispersive system ni Eq. (6), and the dashed curve represents results obtained from the shallow-water system. All variables are non-dimensional. 

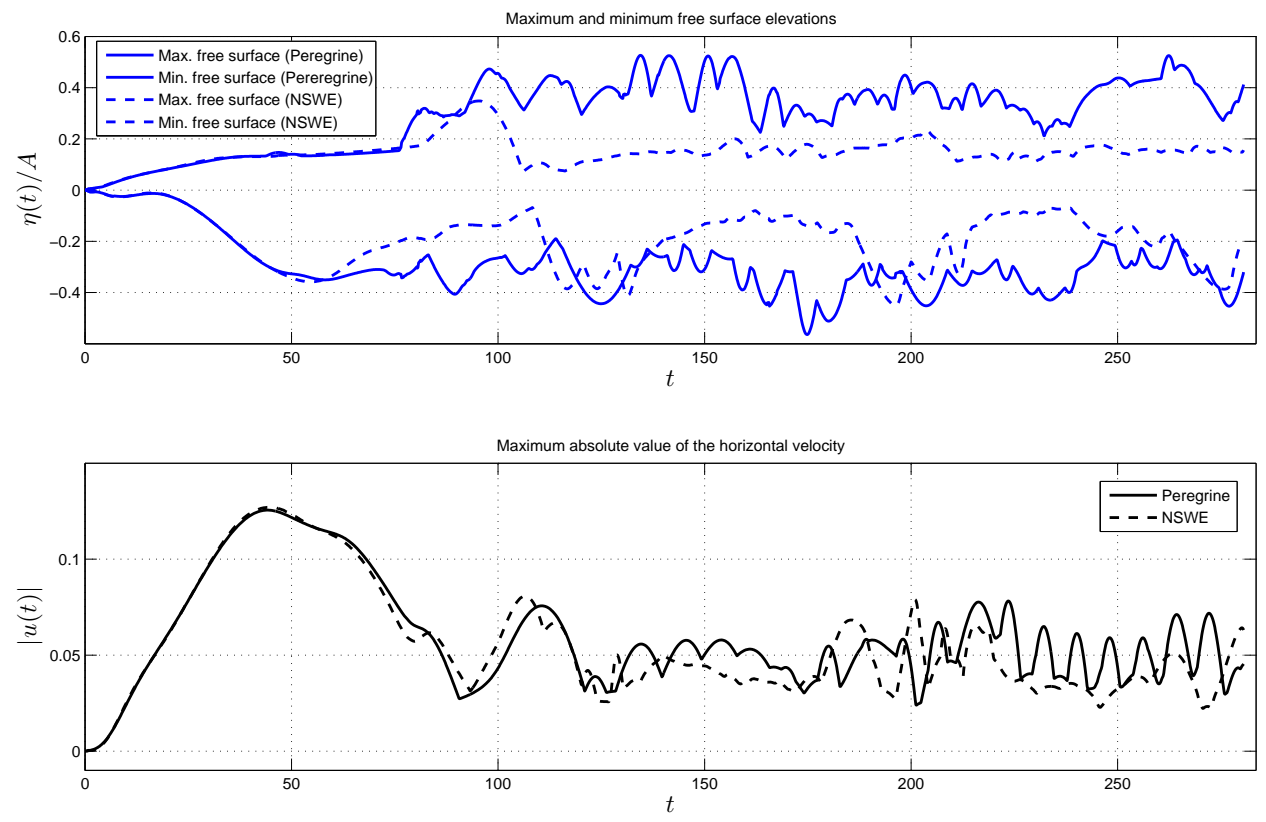

Fig. 13. Maximum and minimum of the surface excursion, and the horizontal velocity as a function of (non-dimensional) time.
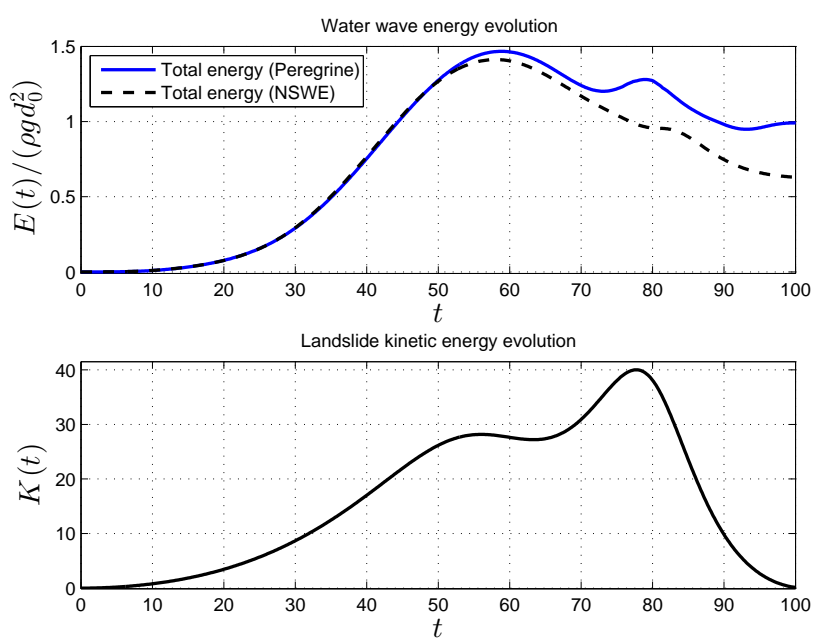

Fig. 14. Development of the wave energy, and the kinetic energy of the landslide as a function of (non-dimensional) time. Note that the kinetic energy of the landslide starts from 0 (all energy is potential) and also ends at 0 (all energy has been dissipated or transferred to the fluid).

dispersive model. The lower panel shows the kinetic energy of the landslide.

We have also computed the Froude number $F r=\frac{v}{\sqrt{g h\left(x_{\mathrm{c}}\right)}}$ during the evolution. Here $v$ is the $x$ component of the velocity of the barycenter of the landslide, $x_{\mathrm{c}}$ the position of the barycenter, and $h\left(x_{\mathrm{c}}\right)$ the corresponding local water depth. This number was always found to be much less than 1 in all
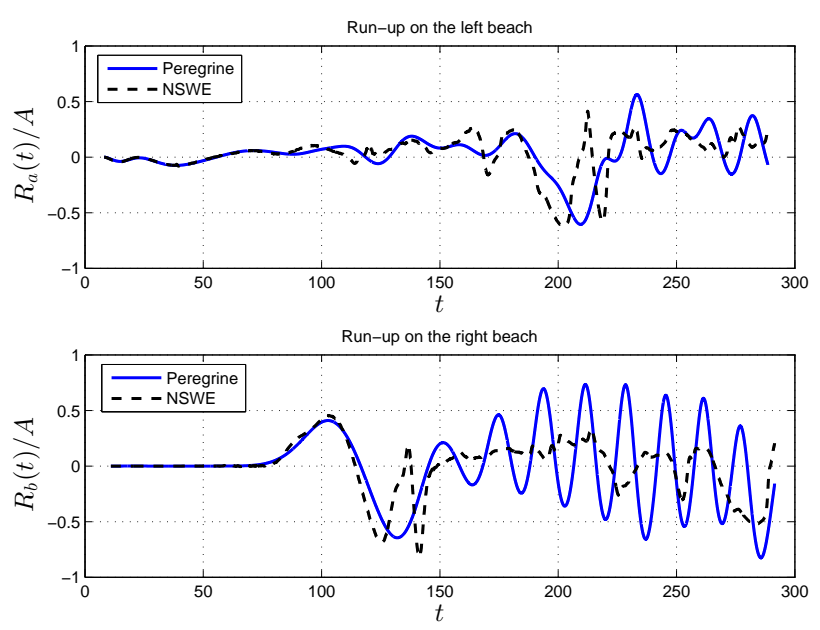

Fig. 15. Run-up on the left and right beach using Eq. (19), computed with the dispersive system (solid curve) and the nonlinear shallowwater system (dashed curve) as a function of (non-dimensional) time.

numerical experiments. The maximum value was generally about 0.5 .

To compute the wave run-up and drawdown, we use exact representations given by Choi et al. (2011) (a similar formula was also derived in Didenkulova and Pelinovsky, 2008). On the right beach, the undisturbed water depth at the edge of the computational domain is $h=3$, and the distance from the computational domain to the shore line is $L=30$. Using the shallow-water wave speed, the travel time of a wave from the 
edge of the computational domain to the shore is computed as

$T=\frac{2 L}{\sqrt{g h}}=2 \sqrt{\frac{L}{g \alpha}}$.

Then the formula for the wave run-up $R$ at the shore reads

$R=\int_{0}^{t-T} \frac{t-\tau}{(t-\tau)^{2}-T^{2}} \cdot \frac{\mathrm{d} \eta}{\mathrm{d} \tau}(x, \tau) \mathrm{d} \tau$

with $x=220$. At the left beach, the undisturbed water depth is $h=1.642$, and the distance to the beach is $L=11.2814$. A similar formula can then be computed for $x=0$.

Figure 15 shows the run-up on the left and right beaches both in the Boussinesq scaling and in the shallow-water theory. While the agreement is fair on the left beach, it appears immediately that the Boussinesq theory predicts a wave runup on the right beach that is much larger (roughly by a factor of two) than the wave run-up according to the shallowwater theory. A possible explanation for this divergence is the nature of the numerical solver when applied to the shallowwater system. In this case, there is continuous numerical dissipation through the handling of hyperbolic wave breaking. Since the waves do not break in the Boussinesq scaling, the dissipation is not present, or at least much smaller. The difference can also be read off from the comparison of the wave energy in the Boussinesq and shallow-water system provided in Fig. 14. It can be seen there that the wave energy in the shallow-water model starts to diverge from the Boussinesq model at non-dimensional time $t=50$. The difference between the two increases continuously until, at the final time, the Boussinesq energy is about $50 \%$ larger than the shallowwater energy. Note that significant run-up in Fig. 15 does not happen until non-dimensional time $t=75$, at which time the energy in the Boussinesq system is already much larger than in the shallow-water system.

In Fig. 16, we have plotted the maximum wave amplitude, the minimum wave amplitude, and the maximum wave runup on the left and right beaches. In comparison to previous studies, such as Grilli and Watts (2005), where an open domain was used, it appears that, in our case, the maximal amplitude, and the run-up have a minimum at $d_{0}$ between 1 and 1.5. In Grilli and Watts (2005), it was found that maximum wave amplitude and run-up (on the left beach) were strictly decreasing functions of $d_{0}$. The phenomenon of rising amplitude and run-up may be accredited to resonant effects that are absent on an open domain (such as an ocean beach), but cannot be neglected for tsunamis generated by landslides in rivers and lakes.

\section{Conclusions}

The influence of an underwater landslide on surface waves in a closed basin has been studied. The key features of the
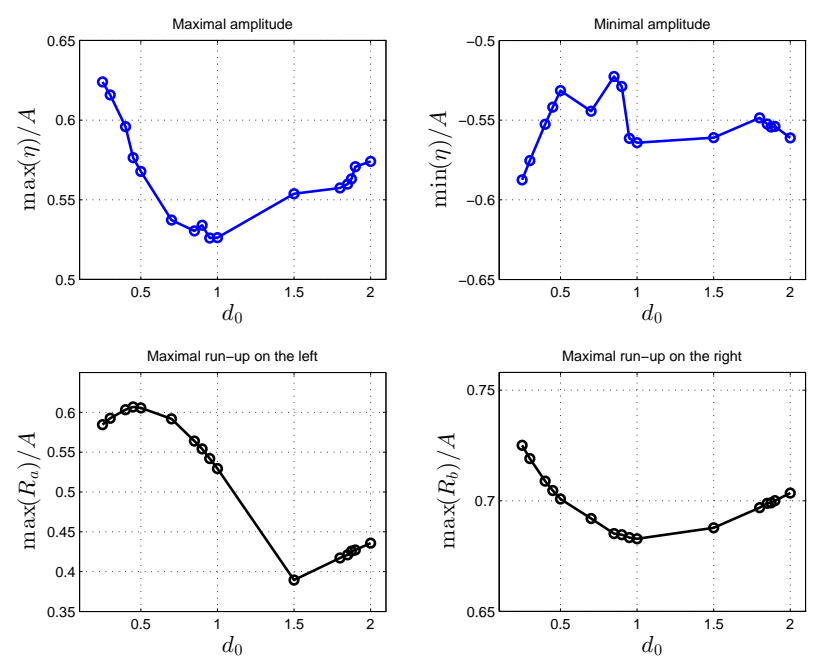

Fig. 16. Maximal and minimal wave amplitude, and the maximum run-up on the left and right beaches as a function of the initial depth of the center of the landslide $d_{0}$.

study have been that the motion of the underwater landslide is determined by integrating a second-order ordinary differential equation derived from first principles of Newtonian mechanics, and that the wave motion is studied in the Boussinesq scaling, which allows for both nonlinear and dispersive effects. The dynamics of the motion of the bottom have been developed following recent work in Khakimzyanov and Shokina (2010). The Boussinesq model that has been utilized here allows for a dynamic bathymetry, and was derived in $\mathrm{Wu}$ (1987). The numerical method used in this paper is an extension of the method put forward in Barth (1994) and Barth and Ohlberger (2004).

The results presented in Sect. 6 clearly show that dispersion may have a strong effect on the run-up and drawdown at the beaches, but it is not clear which of the two models (the shallow-water or the Boussinesq model) paints a more realistic picture of the actual wave conditions. We have no way of quantifying the energy dissipation in the shallow-water solver other than comparing the total wave energy with that of the Boussinesq model. As the difference is rather large, we expect a significant amount of numerical dissipation in the shallow-water simulation. While the shallow-water model simulation might be closer to physical reality where actual damping occurs because of molecular viscosity and fluidstructure interactions, it is likely that the Boussinesq model exhibits a closer resemblance to the Euler equations, which are taken as the basic governing equations in this work.

Of course, this difference could be more or less pronounced depending on the particular case under study. For example, the divergence between the shallow-water theory and the dispersive model is stronger at the right beach than at the left beach. The results also show that a finite domain exhibits different behavior than a half-open domain (such as 
used in Grilli and Watts, 2005) with respect to the dependence of the wave run-up on the initial depth of the landslide. While the run-up is a strictly decreasing function of the initial depth in an open domain, a closed domain appears to exhibit resonant effects, which make the dependence more complex.

Acknowledgements. D. Dutykh would like to thank the University of Bergen for support and hospitality during the preparation of this manuscript. Support from the Agence Nationale de la Recherche under project ANR-08-BLAN-0301-01 (MathOcéan) and from ERC under project ERC-2011-AdG 290562-MULTIWAVE is also gratefully acknowledged.

H. Kalisch acknowledges support of the Research Council of Norway through grant no. NFR 213474/F20.

Edited by: E. Pelinovsky

Reviewed by: M. Brocchini and L. Chubarov

\section{References}

Ali, A. and Kalisch, H.: Energy balance for undular bores, C.R. Mécanique, 338, 67-70, doi:10.1016/j.crme.2010.02.003, 2010.

Ali, A. and Kalisch, H.: Mechanical balance laws for Boussinesq models of surface water waves, J. Nonlinear Sci., 22, 371-398, doi:10.1007/s00332-011-9121-2, 2012.

Bardet, J.-P., Synolakis, C. E., Davies, H. L., Imamura, F., and Okal, E. A.: Landslide Tsunamis: Recent Findings and Research Directions, Pure Appl. Geophys., 160, 1793-1809, doi:10.1007/s00024-003-2406-0, 2003.

Barth, T. J.: Aspects of unstructured grids and finite-volume solvers for the Euler and Navier-Stokes equations, Lecture series - van Karman Institute for Fluid Dynamics, 5, 1-140, 1994.

Barth, T. J. and Ohlberger, M.: Encyclopedia of Computational Mechanics, Volume 1, Fundamentals, chapter Finite Vol. John Wiley and Sons, 2004.

Batchelor, G. K.: An introduction to fluid dynamics, volume 61 of Cambridge mathematical library, Cambridge University Press, Cambridge,

Beisel, S. A., Khakimzyanov, D. G., and Chubarov, L. B.: Surface wave modeling generated by an underwater landslide moving along a nonuniform slope, Comput. Technol., 15, 39-51, 2010.

Beisel, S. A., Chubarov, L. B., Dutykh, D., Khakimzyanov, G., and Shokina, N.: Simulation of surface waves generated by an underwater landslide in a bounded reservoir, Russ. J. Numer. Anal. M., 27, 539-558, doi:10.1515/rnam-2012-0031, 2012.

Bjørkavåg, M. and Kalisch, H.: Wave breaking in Boussinesq models for undular bores, Phys. Lett. A, 375, 1570-1578, doi:10.1016/j.physleta.2011.02.060, 2011.

Bogacki, P. and Shampine, L. F.: A 3(2) pair of Runge-Kutta formulas, Appl. Math. Lett., 2, 321-325, doi:10.1016/08939659(89)90079-7, 1989.

Bona, J. L., Chen, M., and Saut, J.-C.: Boussinesq equations and other systems for small-amplitude long waves in nonlinear dispersive media, I: Derivation and linear theory, J. Nonlinear Sci., 12, 283-318, doi:10.1007/s00332-002-04664doi:10.1007/s00332-002-0466-4, 2002.
Boussinesq, J.: Théorie de l'intumescence liquide appelée onde solitaire ou de translation se propageant dans un canal rectangulaire, C. R. Acad. Sci. I Math., 72, 755-759, 1871.

Briganti, R., Musumeci, R. E., Bellotti, G., Brocchini, M., and Foti, E.: Boussinesq modelling of breaking waves: description of turbulence, J. Geophys. Res.-Oceans, 109, C07015, doi:10.1029/2003JC002065, 2004.

Choi, B. H., Kaistrenko, V., Kim, K. O., Min, B. I., and Pelinovsky, E.: Rapid forecasting of tsunami runup heights from 2-D numerical simulations, Nat. Hazards Earth Syst. Sci., 11, 707714, doi:10.5194/nhess-11-707-2011doi:10.5194/nhess-11-7072011, 2011.

Chubarov, L. B., Eletsky, S., Fedotova, Z., and Khakimzyanov, G. S.: Simulation of surface waves by an underwater landslide, Russ. J. Numer. Anal. M., 20, 425-437, doi:10.1515/156939805775186668, 2005

Chubarov, L. B., Khakimzyanov, G. S., and Shokina, N.: Numerical modelling of surface water waves arising due to movement of underwater landslide on irregular bottom slope, in: Notes on $\mathrm{Nu}-$ merical Fluid Mechanics and Multidisciplinary Design: Computational Science and High Performance Computing IV, SpringerVerlag, Berlin, Heidelberg, vol. 115 edition, 75-91, 2011.

Didenkulova, I. and Pelinovsky, E.: Run-up of long waves on a beach: the influence of the incident wave form, Oceanology, 48 , 1-6, doi:10.1134/S0001437008010013, 2008.

Didenkulova, I., Nikolkina, I., Pelinovsky, E., and Zahibo, N.: Tsunami waves generated by submarine landslides of variable volume: analytical solutions for a basin of variable depth, Nat. Hazards Earth Syst. Sci., 10, 2407-2419, doi:10.5194/nhess-102407-2010doi:10.5194/nhess-10-2407-2010, 2010.

Di Risio, M., Bellotti, G., Panizzo, A., and De Girolamo, P.: Three-dimensional experiments on landslide generated waves at a sloping coast, Coast. Eng., 56, 659-671, doi:10.1016/j.coastaleng.2009.01.009, 2009.

Dutykh, D. and Dias, F.: Energy of tsunami waves generated by bottom motion, Proc. Roy. Soc. A, 465, 725-744, doi:10.1098/rspa.2008.0332, 2009.

Dutykh, D., Dias, F., and Kervella, Y.: Linear theory of wave generation by a moving bottom, C.R. Acad. Sci. I Math., 343, 499504, doi:10.1016/j.crma.2006.09.016, 2006.

Dutykh, D., Katsaounis, T., and Mitsotakis, D.: Dispersive wave runup on non-uniform shores, in: Finite Volumes for Complex Applications VI - Problems \& Perspectives, edited by: Fort, J., Fürst, J., Halama, J., Herbin, R., and Hubert, F., Prague, Springer Berlin Heidelberg, 389-397, 2011a.

Dutykh, D., Katsaounis, T., and Mitsotakis, D.: Finite volume schemes for dispersive wave propagation and runup, J. Comp. Phys., 230, 3035-3061, doi:10.1016/j.jcp.2011.01.003, 2011b.

Dutykh, D., Katsaounis, T., and Mitsotakis, D.: Finite volume methods for unidirectional dispersive wave models, Int. J. Num. Meth. Fluids, 71, 717-736, doi:10.1002/fld.3681, 2013a.

Dutykh, D., Mitsotakis, D., Gardeil, X., and Dias, F.: On the use of the finite fault solution for tsunami generation problems, Theor. Comput. Fluid Dyn., 27, 177-199, doi:10.1007/s00162011-0252-8, 2013b.

Fenton, J.: A ninth-order solution for the solitary wave, J. Fluid Mech., 53, 257-271, doi:10.1017/S002211207200014X, 1972.

Fernández-Nieto, E. D., Bouchut, F., Bresch, D., Castro-Diaz, M. J., and Mangeney, A.: A new Savage-Hutter type model for sub- 
marine avalanches and generated tsunami, J. Comp. Phys., 227, 7720-7754, doi:10.1016/j.jcp.2008.04.039, 2008.

Frigo, M. and Johnson, S. G.: The Design and Implementation of FFTW3, Proc. IEEE, 93, 216-231, 2005.

Fritz, H. M., Kongko, W., Moore, A., McAdoo, B., Goff, J., Harbitz, C. and Uslu, B., Kalligeris, N., Suteja, D., Kalsum, K., Titov, V. V., Gusman, A., Latief, H., Santoso, E., Sujoko, S., Djulkarnaen, D., Sunendar, H., and Synolakis, C.: Extreme runup from the 17 July 2006 Java tsunami, Geophys. Res. Lett., 34, L12602, doi:10.1029/2007GL029404, 2007.

Fuhrman, D. R. and Madsen, P. A.: Tsunami generation, propagation, and run-up with a high-order Boussinesq model, Coast. Eng., 56, 747-758, doi:10.1016/j.coastaleng.2009.02.004, 2009.

Ghidaglia, J.-M., Kumbaro, A. and Le Coq, A.: Une méthode volumes-finis à flux caractéristiques pour la résolution numérique des systèmes hyperboliques de lois de conservation, CR Acad. Sci. I Math., 322, 981-988, 1996.

Ghidaglia, J.-M., Kumbaro, A., and Le Coq, A.: On the numerical solution to two fluid models via cell centered finite volume method, European Journal of Mechanics B/Fluids, Eur. J. Mech. B-Fluid., 20, 841-867, doi:10.1016/S0997-7546(01)01150-5, 2001

Grilli, S. T., Guyenne, P., and Dias, F.: A fully nonlinear model for three-dimensional overturning waves over an arbitrary bottom, Int. J. Num. Methods Fluids, 35, 829-867, doi:10.1002/10970363(20010415)35:7<829::AID-FLD115>3.0.CO;2-2, 2001.

Grilli, S. T. and Watts, P.: Modeling of waves generated by a moving submerged body. Applications to underwater landslides, Eng. Anal. Bound. Elem., 23, 645-656, doi:10.1016/S09557997(99)00021-1, 1999.

Grilli, S. T. and Watts, P.: Tsunami Generation by Submarine Mass Failure. I: Modeling, Experimental Validation, and Sensitivity Analyses, J. Waterw. Port. C. ASCE, 131, 283-297, doi:10.1061/(ASCE)0733-950X(2005)131:6(283), 2005.

Grilli, S. T., Dias, F., Guyenne P., Fochesato, C., and Enet, F.: Progress in fully nonlinear potential flow modeling of 3-D extreme ocean waves, in: Advances in Numerical Simulation of Nonlinear Water Waves, edited by: Ma, Q. W., World Scientific Publishing, 75-128, 2010.

Grimshaw, R.: The solitary wave in water of variable depth, Part 2, J. Fluid Mech., 46, 611-622, doi:10.1017/S0022112071000739doi:10.1017/S0022112071000739, 1971.

Hammack, J.: A note on tsunamis: their generation and propagation in an ocean of uniform depth, J. Fluid Mech., 60, 769-799, doi:10.1017/S0022112073000479, 1973

Harbitz, C. B., Lovholt, F., Pedersen, G., Glimsdal, S., and Masson, D. G.: Mechanisms of tsunami generation by submarine landslides - a short review, Norw. J. Geol., 86, 255-264, 2006.

Harten, A.: ENO schemes with subcell resolution, J. Comp. Phys., 83, 148-184, doi:10.1016/0021-9991(89)90226-X, 1989.

Harten, A. and Osher, S.: Uniformly high-order accurate nonoscillatory schemes, I, SIAM J. Numer. Anal., 24, 279-309, 1987.

Khakimzyanov, G. S. and Shokina, N. Y.: Numerical modelling of surface water waves arising due to a movement of the underwater landslide on an irregular bottom, Comput. Technol., 15, 105-119, 2010.

Kolgan, N. E.: Finite-difference schemes for computation of three dimensional solutions of gas dynamics and calculation of a flow over a body under an angle of attack, Uchenye Zapiski TsaGI [Sci. Notes Central Inst. Aerodyn], 6, 1-6, 1975.

Lynett, P. and Liu, P. L.-F.: A Numerical Study of Submarine Landslide Generated Waves and Runup, Proc. Roy. Soc. A, 458, 2885-2910, doi:10.1098/rspa.2002.0973, 2002.

Nwogu, O.: Alternative form of Boussinesq equations for nearshore wave propagation, J. Waterw. Port. C. ASCE, 119, 618-638, doi:10.1061/(ASCE)0733-950X(1993)119:6(618), 1993.

Okal, E. A.: Normal mode energetics for far-field tsunamis generated by dislocations and landslides, Pure Appl. Geophys., 160, 2189-2221, doi:10.1007/s00024-003-2426-9, 2003.

Okal, E. A. and Synolakis, C. E.: A theoretical comparison of tsunamis from dislocations and landslides, Pure Appl. Geophys., 160, 2177-2188, doi:10.1007/s00024-003-2425-x, 2003.

Pelinovsky, D. E. and Stepanyants, Y. A.: Convergence of Petviashvili's iteration method for numerical approximation of stationary solutions of nonlinear wave equations, SIAM J. Numer. Anal., 42, 1110-1127, doi:10.1137/S0036142902414232, 2004.

Pelinovsky, E. and Poplavsky, A.: Simplified model of tsunami generation by submarine landslides, Phys. Chem. Earth, 21, 13-17, doi:10.1016/S0079-1946(97)00003-7, 1996.

Peregrine, D. H.: Long waves on a beach, J. Fluid Mech., 27, 815827, doi:10.1017/S0022112067002605, 1987.

Poncet, R., Campbell, C., Dias, F., Locat, J., and Mosher, D.: A study of the tsunami effects of two landslides in the St. Lawrence estuary, in: Submarine Mass Movements and Their Consequences, edited by: Mosher, D. C., Shipp, C., Moscardelli, L., Chaytor, J., Baxter, C., Lee, H., and Urgeles, R., 755-764, Springer Verlag, 2010.

Prior, D. B. and Coleman, J. M.: Submarine landslides: geometry and nomenclature, Z. Geomorphologie, 23, 415-426, 1979.

Sammarco, P. and Renzi, E.: Landslide tsunamis propagating along a plane beach, J. Fluid Mech., 598, 107-119, doi:10.1017/S0022112007009731, 2008.

Seo, S. and Liu, Ph. L.-F.: Edge waves generated by the landslide on a sloping beach, Coast. Eng., 73, 133-150, doi:10.1016/j.coastaleng.2012.10.008, 2013.

Shampine, L. F. and Reichelt, M. W.: The MATLAB ODE Suite, SIAM J. Sci. Comput., 18, 1-22, doi:10.1137/S1064827594276424, 1997

Shokin, Yu. I., Fedotova, Z., Khakimzyanov, G. S., Chubarov, L. B., and Beisel, S. A.: Modelling surface waves generated by a moving landslide with allowance for vertical flow structure, Russ. J. Numer. Anal. M., 22, 63-85, doi:10.1515/RNAM.2007.22.1.63, 2007.

Söderlind, G.: Digital filters in adaptive time-stepping, ACM Transactions on Mathematical Software, 29, 1-26, 2003.

Söderlind, G. and Wang, L.: Adaptive time-stepping and computational stability, J. Comp. Appl. Math., 185, 225-243, doi:10.1016/j.cam.2005.03.008, 2006.

Tadepalli, S. and Synolakis, C. E.: Model for the leading waves of tsunamis, Phys. Rev. Lett., 77, 2141-2144, doi:10.1103/PhysRevLett.77.2141, 1996.

Tinti, S., and Bortolucci, E.: Energy of Water Waves Induced by Submarine Landslides, Pure Appl. Geophys., 157, 281-318, doi:10.1007/s000240050001, 2000.

Tinti, S., Bortolucci, E., and Chiavettieri, C.: Tsunami Excitation by Submarine Slides in Shallow-water Approximation, Pure Appl. Geophys., 158, 759-797, doi:10.1007/PL00001203, 2001. 
van Leer, B.: Towards the ultimate conservative difference scheme V: a second order sequel to Godunov' method, J. Comp. Phys., 32, 101-136, doi:10.1016/0021-9991(79)90145-1, 1979.

van Leer, B.: Upwind and High-Resolution Methods for Compressible Flow: From Donor Cell to Residual-Distribution Schemes, Comm. Comp. Phys., 1, 192-206, 2006.

Watts, P., Imamura, F., and Grilli, S. T.: Comparing model simulations of three benchmark tsunami generation cases, Sci. Tsunami Hazards, 18, 107-123, 2000.

Watts, P., Grilli, S. T., Kirby, J. T., Fryer, G. J., and Tappin, D. R.: Landslide tsunami case studies using a Boussinesq model and a fully nonlinear tsunami generation model, Nat. Hazards Earth Syst. Sci., 3, 391-402, doi:10.5194/nhess-3-3912003doi:10.5194/nhess-3-391-2003, 2003.
Whitham, G. B.: Linear and nonlinear waves, John Wiley \& Sons Inc., New York, 1974.

$\mathrm{Wu}$, T. Y.-T.: Generation of upstream advancing solitons by moving disturbances, J. Fluid Mech., 184, 75-99, doi:10.1017/S0022112087002817, 1987.

Xing, Y. and Shu, C.-W.: High order finite difference WENO schemes with the exact conservation property for the shallow water equations, J. Comp. Phys., 208, 206-227, doi:10.1016/j.jcp.2005.02.006, 2005. 